\title{
Nonequilibrium thermodynamics of interfaces using classical density functional theory
}

\author{
Eivind Johannessen, ${ }^{1}$ Joachim Gross, ${ }^{2}$ and Dick Bedeaux ${ }^{1,2, a)}$ \\ ${ }^{1}$ Department of Chemistry, Norwegian University of Science and Technology, N-7491 Trondheim, Norway \\ ${ }^{2}$ Engineering Thermodynamics, Delft University of Technology, Leeghwaterstraat 44, \\ 2628 CA Delft, The Netherlands
}

(Received 16 May 2008; accepted 7 October 2008; published online 12 November 2008)

\begin{abstract}
A vapor-liquid interface introduces resistivities for mass and heat transfer. These resistivities have recently been determined from molecular simulations, as well as theoretically using the van der Waals square gradient model. This model, however, does not allow for direct quantitative comparison to experiment or results from molecular simulations. The classical density functional theory is used here in order to determine the equilibrium profiles of vapor-liquid interfaces. Equilibrium profiles are sufficient in the framework of nonequilibrium thermodynamics for determining the interfacial resistivities. The interfacial resistivities for heat transfer, for mass transfer, and for the coupling of heat and mass transfer can all be related to only one local thermal resistivity. This is done with integral relations for the interfacial resistivities. All interfacial resistivities can be consistently described in their temperature behavior with good accuracy. () 2008 American Institute of Physics. [DOI: 10.1063/1.3009182]
\end{abstract}

\section{INTRODUCTION}

In evaporation and condensation, mass is transferred across the liquid-vapor interface. In order to supply or to carry away the corresponding latent heat, heat is flowing either towards or away from the surface. In the modeling of these processes, it is usually assumed that there is equilibrium at the interface. ${ }^{1}$ The validity of this assumption is questionable. Experiments have shown that there are temperature jumps across the interface of several degrees, see Badam et $a l^{2}{ }^{2}$ and references therein. It is therefore essential to describe the dynamics at the interface correctly in order to understand and predict evaporation and condensation processes better.

We consider stationary evaporation and condensation of a one-component fluid. All fluxes and gradients are normal to the interface (along the $z$ axis). The liquid and the vapor are on the left- and on the right-hand sides, respectively. We use the equimolar surface as the dividing surface. Nonequilibrium thermodynamics for surfaces ${ }^{3,4}$ describes the transfer of heat and particles for evaporation and condensation., ${ }^{5,6}$ This description finds that the thermodynamic driving forces are linear functions of the heat and particle fluxes through the surface. Using the measurable heat flux, $J_{q}^{\prime 1}$, on the liquid side and the particle flux $J$, one finds

$$
\begin{aligned}
& \frac{1}{T^{g}}-\frac{1}{T^{l}}=R_{q q}^{l} J_{q}^{\prime l}+R_{q \mu}^{l} J, \\
& \frac{\mu^{g}\left(T^{g}\right)-\mu^{l}\left(T^{g}\right)}{T^{g}}=R_{\mu q}^{l} J_{q}^{\prime l}+R_{\mu \mu}^{l} J,
\end{aligned}
$$

\footnotetext{
${ }^{a)}$ Author to whom correspondence should be addressed. Electronic mail: dick.bedeaux@chem.ntnu.no.
}

where the $R$ 's are the interfacial resistivities for the liquid side. Here $T^{1}, \mu^{1}$ and $T^{g}, \mu^{g}$ are the temperature and chemical potential on the liquid and the vapor sides of the surface. Using the measurable heat flux on the vapor side, $J_{q}^{\prime g}$, and the particle flux, one analogously finds

$$
\begin{aligned}
& \frac{1}{T^{g}}-\frac{1}{T^{l}}=R_{q q}^{g} J_{q}^{\prime g}+R_{q \mu}^{g} J, \\
& \frac{\mu^{g}\left(T^{l}\right)-\mu^{l}\left(T^{l}\right)}{T^{l}}=R_{\mu q}^{g} J_{q}^{\prime g}+R_{\mu \mu}^{g} J,
\end{aligned}
$$

where the $R$ 's are now the interfacial resistivities for the vapor side. The particle flux is constant through the interfacial region, while the measurable heat fluxes differ on both sides. It is important to take the coupling between the heat and mass transfer processes into account. It has been shown that neglecting the coupling leads to a violation of the second law. ${ }^{7}$ We shall refer to the linear coefficients in this relation as interfacial transfer coefficients or interfacial resistivities.

The interfacial resistivities for heat and mass transfer in a one-component system have been calculated using kinetic theory, ${ }^{5-8-11}$ nonequilibrium molecular dynamics (NEMD) ${ }^{12-15}$ experimental results, ${ }^{16,17,2}$ and using the nonequilibrium van der Waals square gradient model. ${ }^{18-20}$ The NEMD simulations are (computer) time consuming. It is difficult to obtain all four interfacial resistivities involved. The experiments lead to values which are more than an order of magnitude larger than the values predicted both by kinetic theory and by using NEMD simulations. The nonequilibrium van der Waals square gradient model contains adjustable parameters and is therefore useful to obtain relative values but not the absolute value.

In this paper, we will use integral relations, which were derived in an earlier paper, ${ }^{21}$ to calculate the interfacial re- 
sistivities. These integral relations were found from a continuous description in which the measurable heat flux, $J_{q}^{\prime}(z)$, as a function of the position is given by

$$
\frac{d}{d z}\left(\frac{1}{T(z)}\right)=r_{q q}(z) J_{q}^{\prime}(z),
$$

where $T(z)$ is the temperature along the $z$ axis. Furthermore, $r_{q q}(z)$ is the local thermal resistivity. In NEMD simulations, one obtains this relation and the resulting thermal resistivity. It is sufficient to consider only a heat flux; a particle flux is not needed. In the interfacial region the local thermal resistivity deviates from the extrapolated values on both sides of the surface. This is the reason why it is necessary to ascribe finite resistivities for heat and particle transfer in Eqs. (1) and (2). In the NEMD simulations, ${ }^{22,23}$ it is found to be appropriate to use a film with a finite thickness between the positions $z^{s, g}$ and $z^{s, l}$ as the interfacial region. We will discuss how these positions are chosen below. The measurable heat fluxes on the liquid and the vapor sides are then identified with $J_{q}^{\prime l}=J_{q}^{\prime}\left(z^{s, l}\right)$ and $J_{q}^{\prime g}=J_{q}^{\prime}\left(z^{s, g}\right)$. The temperatures and chemical potentials in the thermodynamic forces are similarly calculated from the continuous profiles at $z^{s, g}$ and $z^{s, l}$.

The integral relations derived for the interfacial resistivities are $^{21}$

$$
\begin{aligned}
& R_{q q}^{l}=\int_{z^{s, l}}^{z^{s, g}} r_{q q}(z) d z, \\
& R_{q \mu}^{l}=R_{\mu q}^{l}=\int_{z^{s, l}}^{z^{s, g}} r_{q q}(z)\left[h\left(z^{s, l}\right)-h(z)\right] d z, \\
& R_{\mu \mu}^{l}=\int_{z^{s, l}}^{z^{s, g}} r_{q q}(z)\left[h\left(z^{s, l}\right)-h(z)\right]^{2} d z,
\end{aligned}
$$

on the liquid side, and

$$
\begin{aligned}
& R_{q q}^{g}=R_{q q}^{l}=\int_{z^{s, l}}^{z^{s, g}} r_{q q}(z) d z, \\
& R_{q \mu}^{g}=R_{\mu q}^{g}=\int_{z^{s, l}}^{z^{s, g}} r_{q q}(z)\left[h\left(z^{s, g}\right)-h(z)\right] d z, \\
& R_{\mu \mu}^{g}=\int_{z^{s, l}}^{z^{s, g}} r_{q q}(z)\left[h\left(z^{s, g}\right)-h(z)\right]^{2} d z
\end{aligned}
$$

on the vapor side. Here, $h(z)$ is the molar enthalpy at position $z$. The expressions for the interfacial resistivities clearly show that these resistivities depend on the choice of $z^{s, g}$ and $z^{s, l}$. If one chooses the interface film thicker, the resistivities increase. In the NEMD simulations, ${ }^{12-15}$ a criterion is formulated such that these positions are close to the interfacial region. There remains nevertheless some ambiguity in that one may choose somewhat different criteria to characterize "close." This dependence is not unlike the dependence of excess densities on the choice of the dividing surface, and we will return to this point below.

In the NEMD simulations, it is reasonably straightforward to calculate the local resistivity for heat transfer $r_{q q}$ and the enthalpy density. We were thus able to verify the validity of the integral relations directly, see Refs. 22 and 23. For $n$-octane molecules we had not been able to do simulations with a finite mass flux, ${ }^{13}$ as these were too time consuming. We were therefore unable to calculate $R_{\mu \mu}^{g}$ and $R_{22}^{l}$ directly. This could be done using the integral relations. ${ }^{22}$

The integral relations show that the resistivities for heat and mass transfer for the whole surface can be calculated once the local thermal resistivity $r_{q q}(z)$ and the enthalpy profile are known. For the thermal resistivity, we use an expression for the local heat transfer resistance that was given and motivated for the van der Waals square gradient model, ${ }^{18-20}$

$$
\begin{aligned}
r_{q q}(z)= & r_{q q, \mathrm{gas}}^{\mathrm{Coex}}[T(z)]+\left\{r_{q q, \mathrm{liq}}^{\mathrm{Coex}}[T(z)]-r_{q q, \mathrm{gas}}^{\mathrm{Coex}}[T(z)]\right\} \\
& \times\left(\frac{\rho(z)-\rho_{\mathrm{gas}}^{\mathrm{Cox}}[T(z)]}{\rho_{\mathrm{liq}}^{\operatorname{Coex}}[T(z)]-\rho_{\mathrm{gas}}^{\operatorname{Coex}}[T(z)]}\right) \\
& +\alpha\left(\frac{\rho_{\mathrm{liq}}^{\operatorname{Coex}}[T(z)]}{\rho(z)}\right)^{\beta}\left(\frac{d \rho(z)}{d z}\right)^{2} .
\end{aligned}
$$

The first two terms in the local heat transfer resistance give a linear interpolation between the resistivities in the bulk liquid and bulk vapor along the coexistence line. These resistivities are the inverse of the thermal conductivities, $\lambda$, divided by the temperature squared, $\lambda=\left[r_{q q} T^{2}\right]^{-1}$. The last term in the local heat transfer resistance gives an additional contribution in the interfacial region. In the square gradient theory for the interface, the local heat transfer resistance in this region may not only depend on the local density and temperature, but may also contain a contribution proportional to the square gradient as well as a contribution proportional to the second derivative of the density. As the last one is not systematically positive we used only the term proportional to the square gradient. The factor $\left(\rho_{\text {liq }}^{\text {Coex }}(T(z)) / \rho(z)\right)^{\beta}$ for $\beta>0$ is large on the vapor side and of the order 1 on the liquid side. We shall call $\beta$ the asymmetry parameter. Increasing $\beta$ shifts the peak of the thermal resistivity further towards the vapor side of the surface and increases the integrated size of this contribution. NEMD simulations for the local heat transfer resistance ${ }^{13}$ indicated the need for such a prefactor. The integrated size of this contribution can furthermore be increased or decreased by increasing or decreasing $\alpha$, which is a positive constant. We will call $\alpha$ the overall size parameter. As there is no a priori way to estimate $\alpha$ and $\beta$, these parameters will be fitted to the NEMD data in question. Both $\alpha$ and $\beta$ are phenomenological coefficients, which may in principal depend on the local density and temperature. The reason to use the form of the third term given is that a prediction of the value of the interfacial resistivity matrices could be given, which compared well with the results of the NEMD simulations for all temperatures between the triple and the critical points, using values of $\alpha$ and $\beta$ which were indepent of the local density and temperature.

In the integrand in Eq. (4) one should use the equilibrium profile for the constant temperature (chemical potential and normal pressure) considered. The reason for this is that Eqs. (1) and (2) give the linear response of the temperature and chemical potential differences across the surface to finite heat and/or mass fluxes. As the right hand side of these equa- 
tions are linear in these fluxes, the resistivity matrices do not and should not depend on these fluxes. They should therefore be calculated using the equilibrium profiles. One has the analogous situation in the Green-Kubo relations for transport coefficients, which contain integrals over unequal time equilibrium correlation functions.

The further outline of this paper is as follows. In the second section, we will clarify how we use density functional theory (DFT) to obtain the equilibrium properties of the coexistent phases. Lennard-Jones (LJ) fluids are thereby considered in Sec. III so that the results can be compared to molecular simulation data and to experimental data for argon. Exact expressions for the total Helmholtz energy and the chemical potential are given as integrals over the pair correlation function in Sec. IV. In the fifth section, these expressions are approximated applying a perturbation theory, given as an expansion to second order in the attractive contribution to the potential. The resulting expressions for the thermodynamic variables are given in Sec. VI and contain only the pair correlation function for the hard sphere potential. The numerical calculations are explained in Sec. VII, and the numerical results are presented in Sec. VIII.

\section{INTRODUCTION TO DFT}

The classical DFT is based on a theorem ${ }^{24}$ stating (in the context of classical fluids) that for a system in equilibrium at given temperature $T$, volume $V$, and chemical potentials $\mu_{i}$, an external potential uniquely relates to a distribution in single particle density. For fluids with spherical intermolecular potentials, an orientational distribution does not need to be considered. The grand potential is defined in terms of $\left(T, V, \mu_{i}\right)$ as the natural variables and a solution of the equilibrium density profile $\rho(\mathbf{r})$ (in $\mathrm{m}^{-3}$ ) is obtained by minimizing the grand potential $\Omega$. A prominent publication on the classical DFT is due to Evans, ${ }^{25}$ and a comprehensive review on recent applications is available by $\mathrm{Wu}^{26}$

The grand potential is given in terms of the Helmholtz energy $F$ and the number of particles $N$ by

$$
\Omega=F-\mu N .
$$

The number of particles is given by

$$
N=\int \rho(\mathbf{r}) d \mathbf{r},
$$

where the integral is over the volume in which the system is enclosed. The equilibrium profile of the density is the one that minimizes the grand potential, so that

$$
\left.\frac{\delta \Omega}{\delta \rho(\mathbf{r})}\right|_{\rho(r)=\rho_{\mathrm{eq}}(\mathbf{r})}=\left.\frac{\delta F}{\delta \rho(\mathbf{r})}\right|_{\rho(\mathbf{r})=\rho_{\mathrm{eq}}(\mathbf{r})}-\mu=0,
$$

where we used $\mu$ as the constant. In this study, we relate the nonequilibrium behavior of interfaces to the properties of an interface in equilibrium. We will thus solely be concerned with the density profile $\rho_{\text {eq }}(\mathbf{r})$, and we omit the index "eq" in the following for brevity. With $\delta / \delta \rho(\mathbf{r})$ we indicate the functional derivative with respect to the density in $\mathbf{r}$. Furthermore, $\Omega, F$, and $N$ are functionals of the density distribution. $^{25,27}$ The Helmholtz energy can be decomposed in the Helmholtz energy of an ideal gas and an excess part as

$$
F[\rho(\mathbf{r}) ; T]=F^{\mathrm{id}}[\rho(\mathbf{r}) ; T]+F^{\operatorname{ex}}[\rho(\mathbf{r}) ; T],
$$

where the ideal gas contribution is known exactly, with

$$
F^{\mathrm{id}}[\rho(\mathbf{r}) ; T]=k_{B} T \int d \mathbf{r} \rho(\mathbf{r})\left[\ln \left(\rho(\mathbf{r}) \Lambda^{3}\right)-1\right],
$$

where $\Lambda$ denotes the de Broglie wavelength. The notation $F^{\mathrm{id}}[\rho(\mathbf{r}) ; T]$ indicates that $F^{\mathrm{id}}$ is a functional of the whole density distribution, and a regular function of the temperature, which is constant in equilibrium.

\section{MOLECULAR MODELS}

For getting to an expression of the Helmholtz energy, it is instrumental to decompose the intermolecular pair potential in a repulsive part $u^{0}$ and in an attractive contribution $u^{a}$ as

$$
u\left(\mathbf{r}_{1}, \mathbf{r}_{2}\right)=u^{0}\left(\mathbf{r}_{1}, \mathbf{r}_{2}\right)+u^{a}\left(\mathbf{r}_{1}, \mathbf{r}_{2}\right) .
$$

We refer to $u^{0}$ as the reference potential. Two molecular models are considered here: on the one hand the cutoff and shifted LJ potential and on the other hand the LJ spline potential. These two molecular models were selected because molecular simulation data of nonequilibrium interfaces are available for both types of fluids. In both cases, the total molecular interaction is assumed to be the sum of pair potentials. The cutoff and shifted LJ potential is given as

$$
\begin{aligned}
u\left(\mathbf{r}_{1}, \mathbf{r}_{2}\right)= & 4 \varepsilon\left[\left(\frac{\sigma}{r_{12}}\right)^{12}-\left(\frac{\sigma}{r_{12}}\right)^{6}-\left(\frac{\sigma}{r_{c}}\right)^{12}+\left(\frac{\sigma}{r_{c}}\right)^{6}\right] \\
& \times \theta\left(r_{c}-r_{12}\right),
\end{aligned}
$$

where $r_{12} \equiv\left|\mathbf{r}_{1}-\mathbf{r}_{2}\right|$, and where $\theta(s)$ is the Heaviside function, which is zero when $s<0$ and one when $s>0$. The minimum of the potential is located at $r_{\min }=2^{1 / 6} \sigma$. Vrabec et al. ${ }^{28}$ provided molecular simulations for this molecular model. We split up this potential, following the prescription of Weeks et $a .^{29}$ as

$$
\begin{aligned}
u^{0}\left(\mathbf{r}_{1}, \mathbf{r}_{2}\right) \equiv & \varepsilon\left[1+4\left(\frac{\sigma}{r_{12}}\right)^{12}-4\left(\frac{\sigma}{r_{12}}\right)^{6}\right] \theta\left(r_{\min }-r_{12}\right) \\
u^{a}\left(\mathbf{r}_{1}, \mathbf{r}_{2}\right) \equiv & -\varepsilon \theta\left(r_{\min }-r_{12}\right)+4 \varepsilon\left[\left(\frac{\sigma}{r_{12}}\right)^{12}\right. \\
& \left.-\left(\frac{\sigma}{r_{12}}\right)^{6}\right] \theta\left(r_{12}-r_{\min }\right) \theta\left(r_{c}-r_{12}\right) \\
& -4 \varepsilon\left[\left(\frac{\sigma}{r_{c}}\right)^{12}-\left(\frac{\sigma}{r_{c}}\right)^{6}\right] \theta\left(r_{c}-r_{12}\right)
\end{aligned}
$$

Secondly, we consider the LJ spline potential

$$
u\left(r_{i j}\right)=\left\{\begin{array}{cc}
4 \varepsilon\left[\left(\sigma / r_{i j}\right)^{12}-\left(\sigma / r_{i j}\right)^{6}\right], & 0<r_{i j}<r_{s} \\
a\left(r_{i j}-r_{c}\right)^{2}+b\left(r_{i j}-r_{c}\right)^{3}, & r_{s}<r_{i j}<r_{c} \\
0, & r_{c}<r_{i j},
\end{array}\right.
$$

where $r_{c}=2.5 \sigma$ is the truncated distance and $r_{s}=(48 / 67) r_{c}$. The constants $a$ and $b$ were chosen so that the potential and its derivative are continuous at $r_{s}$. The potential and its de- 
rivative are also continuous in $r_{c}$. In the NEMD simulations, $r_{c}=(26 / 7)^{1 / 6} \sigma \simeq 1.24 \sigma$ was used by Røsjorde and co-workers ${ }^{30,12,14}$ and $r_{c}=2.5 \sigma$ was used by Ge et al. ${ }^{15}$ The prescription of Weeks et al. ${ }^{29}$ gives for the attractive part of the potential

$$
\begin{aligned}
u^{a}\left(\mathbf{r}_{1}, \mathbf{r}_{2}\right) \equiv & -\varepsilon \theta\left(r_{\min }-r_{12}\right)+4 \varepsilon\left[\left(\frac{\sigma}{r_{12}}\right)^{12}-\left(\frac{\sigma}{r_{12}}\right)^{6}\right] \\
& \times \theta\left(r_{12}-r_{\min }\right) \theta\left(r_{s}-r_{12}\right)+\left[a\left(r_{i j}-r_{c}\right)^{2}\right. \\
& \left.+b\left(r_{i j}-r_{c}\right)^{3}\right] \theta\left(r_{12}-r_{s}\right) \theta\left(r_{c}-r_{12}\right) .
\end{aligned}
$$

The repulsive part is the same as for the cutoff shifted $\mathrm{LJ}$ case.

In the analysis, the repulsive potential is replaced by a hard sphere potential. We determine the temperature dependent diameter $d$ using the Barker-Henderson ${ }^{31}$ prescription

$$
d(T) \equiv \int_{0}^{r_{\min }}\left[1-\exp \left(-\frac{u^{0}(r)}{k_{B} T}\right)\right] d r .
$$

This choice has the advantage that it does not depend on the density, like the diameter introduced by Weeks et al. ${ }^{29}$ For both the cutoff shifted LJ and the spline potentials defined above, the hard sphere diameter is the same.

For the further theory, it is also needed to introduce the potential

$$
\begin{aligned}
u_{\lambda}\left(\mathbf{r}_{1}, \mathbf{r}_{2}\right) & =u^{0}\left(\mathbf{r}_{1}, \mathbf{r}_{2}\right)+\lambda u^{a}\left(\mathbf{r}_{1}, \mathbf{r}_{2}\right) \\
& =u^{0}\left(\mathbf{r}_{1}, \mathbf{r}_{2}\right)+\lambda\left[u\left(\mathbf{r}_{1}, \mathbf{r}_{2}\right)-u^{0}\left(\mathbf{r}_{1}, \mathbf{r}_{2}\right)\right],
\end{aligned}
$$

where $0 \leqslant \lambda \leqslant 1$. In this form of the potential, one can reduce the attractive contribution to zero by reducing $\lambda$. For $\lambda=0$, the interaction reduces to the reference potential which is purely repulsive.

\section{HELMHOLTZ ENERGY FUNCTIONAL}

In order to make use of Eq. (9), we need an expression for the Helmholtz energy. The Helmholtz energy $F^{0}$ of the reference fluid, described by the purely repulsive potential $u^{0}$ is easier to obtain than the total Helmholtz energy. The reason for this is that the dimensionless Helmholtz energy is then a function of the dimensionless density only. For the homogeneous reference fluid, one may calculate the Helmholtz energy density (in $\left.\mathrm{J} \mathrm{m}^{-3}\right), f^{0}(\rho, T)$, as a function of the density and temperature. A good approximation is given using results for the hard sphere model. In this paper, we shall use the Carnahan-Starling approximation for the Helmholtz energy density of the hard sphere fluid, ${ }^{32}$ with

$$
f^{0}(\rho, T)=k_{B} T \rho\left[\ln \left(\rho \Lambda^{3}\right)-1+\eta(4-3 \eta)(1-\eta)^{-2}\right],
$$

where

$$
\eta(\rho, T) \equiv \frac{1}{6} \pi d^{3}(T) \rho .
$$

It is noteworthy that Eq. (19) already includes the ideal gas contribution Eq. (11) through the term $\ln \left(\rho \Lambda^{3}\right)-1$. For the Helmholtz energy of the reference fluid, we use the local density approximation (LDA) which gives the value as an integral of the reference Helmholtz energy density over the system at the local density as

$$
F^{0}[\rho(\mathbf{r}) ; T]=\int d \mathbf{r} f^{0}(\rho(\mathbf{r}), T) .
$$

In the description of the liquid-vapor interface, the LDA works well for the contribution to the Helmholtz energy due to repulsive interaction. ${ }^{25,27}$ For cases where local density fluctuations occur, say at solid-liquid interfaces, the LDA fails, while the fundamental measure theory ${ }^{33-35}$ has been shown to be very adequate.

The attractive interaction results in nonlocal contributions. For pairwise interactions, the Helmholtz energy can then be written as ${ }^{25,27}$

$$
\begin{aligned}
F[\rho(\mathbf{r}) ; T]= & F^{0}[\rho(\mathbf{r}) ; T]+\frac{1}{2} \int_{0}^{1} d \lambda \iint d \mathbf{r}_{1} d \mathbf{r}_{2} g_{\lambda}\left(\mathbf{r}_{1}, \mathbf{r}_{2}\right) \\
& \times \rho\left(\mathbf{r}_{1}\right) \rho\left(\mathbf{r}_{2}\right) u^{a}\left(\mathbf{r}_{1}, \mathbf{r}_{2}\right),
\end{aligned}
$$

where $g_{\lambda}\left(\mathbf{r}_{1}, \mathbf{r}_{2}\right)$ is the pair distribution function for an (inhomogeneous) fluid with the intermolecular potential $u_{\lambda}$. This pair distribution function is symmetric for the interchange of $\mathbf{r}_{1}$ and $\mathbf{r}_{2}$, i.e., $g_{\lambda}\left(\mathbf{r}_{1}, \mathbf{r}_{2}\right)=g_{\lambda}\left(\mathbf{r}_{2}, \mathbf{r}_{1}\right)$. Substituting Eq. (22) into Eq. (9) one finds, using this symmetry, the following equation for the chemical potential:

$$
\begin{aligned}
\mu= & \frac{\partial f^{0}\left(\rho_{\mathrm{eq}}(\mathbf{r}), T\right)}{\partial \rho_{\mathrm{eq}}(\mathbf{r})}+\int_{0}^{1} d \lambda \int d \mathbf{r}^{\prime}\left[g_{\lambda}\left(\mathbf{r}, \mathbf{r}^{\prime}\right)\right. \\
& \left.+\rho_{\mathrm{eq}}(\mathbf{r}) \frac{\partial g_{\lambda}\left(\mathbf{r}, \mathbf{r}^{\prime}\right)}{\partial \rho_{\mathrm{eq}}(\mathbf{r})}\right] \rho_{\mathrm{eq}}\left(\mathbf{r}^{\prime}\right) u^{a}\left(\mathbf{r}, \mathbf{r}^{\prime}\right) .
\end{aligned}
$$

When $f^{0}$ and $g_{\lambda}$ are known, Eq. (23) makes it possible to calculate the equilibrium density profile. For both these quantities, one needs approximations in order to make further progress. For $f^{0}$, we will use the Carnahan-Starling approximation Eq. (19). We discuss the pair distribution function in the next section.

\section{EXPANDING AROUND THE HARD SPHERE PAIR DISTRIBUTION FUNCTION}

Not much is known about the pair distribution function in the interfacial region. In this region, the density changes rapidly, which makes the calculation of the pair correlation function difficult. We shall assume that one may use the pair correlation function of the homogeneous fluid at the average of the densities in $\mathbf{r}_{1}$ and $\mathbf{r}_{2}$,

$$
\bar{\rho}_{12}=\frac{1}{2}\left[\rho\left(\mathbf{r}_{1}\right)+\rho\left(\mathbf{r}_{2}\right)\right] .
$$

An expansion of Eq. (22) around the reference fluid $(\lambda$ $=0$ ) leads to a first order term that is written in terms of the reference pair distribution function $g_{0}\left(\mathbf{r}_{2}, \mathbf{r}_{2}, \bar{\rho}_{12}\right)$, while the second order term involves three- and four-body correlation functions that are not easily accessible. ${ }^{36,37}$ We follow the prescription of Barker and Henderson, who, in their perturbation theory, ${ }^{38,31}$ have suggested an approximation of the second order term involving only the pair distribution function. Higher order terms are not accounted for. The Helmholtz energy is then approximated as 


$$
\begin{aligned}
F[\rho(\mathbf{r}) ; T]= & F^{0}[\rho(\mathbf{r}) ; T]+\frac{1}{2} \iint d \mathbf{r}_{1} d \mathbf{r}_{2} g_{0}\left(\mathbf{r}_{1}, \mathbf{r}_{2} ; \bar{\rho}_{12}\right) \rho\left(\mathbf{r}_{1}\right) \rho\left(\mathbf{r}_{2}\right) u^{a}\left(\mathbf{r}_{1}, \mathbf{r}_{2}\right)-\frac{1}{4} \iint d \mathbf{r}_{1} d \mathbf{r}_{2} \frac{\partial \rho\left(\mathbf{r}_{1}\right)}{\partial p_{0}} g_{0}\left(\mathbf{r}_{1}, \mathbf{r}_{2} ; \bar{\rho}_{12}\right) \rho\left(\mathbf{r}_{1}\right) \rho\left(\mathbf{r}_{2}\right) \\
& \times\left[u^{a}\left(\mathbf{r}_{1}, \mathbf{r}_{2}\right)\right]^{2}=F^{0}[\rho(\mathbf{r}) ; T]+\frac{1}{2} \iint d \mathbf{r}_{1} d \mathbf{r}_{2} g_{0}\left(\mathbf{r}_{1}, \mathbf{r}_{2} ; \bar{\rho}_{12}\right) \rho\left(\mathbf{r}_{1}\right) \rho\left(\mathbf{r}_{2}\right) u^{a}\left(\mathbf{r}_{1}, \mathbf{r}_{2}\right) \\
& -\frac{1}{4} \iint d \mathbf{r}_{1} d \mathbf{r}_{2} \frac{\partial \bar{\rho}_{12}}{\partial p_{0}} g_{0}\left(\mathbf{r}_{1}, \mathbf{r}_{2} ; \bar{\rho}_{12}\right) \rho\left(\mathbf{r}_{1}\right) \rho\left(\mathbf{r}_{2}\right)\left[u^{a}\left(\mathbf{r}_{1}, \mathbf{r}_{2}\right)\right]^{2} .
\end{aligned}
$$

In the last equality, we used the symmetry of $g_{0}\left(\mathbf{r}_{1}, \mathbf{r}_{2} ; \bar{\rho}_{12}\right)$ and $u^{a}\left(\mathbf{r}_{1}, \mathbf{r}_{2}\right)$ for the interchange of $\mathbf{r}_{1}$ and $\mathbf{r}_{2}$. By substituting Eq. (25) into Eq. (9), one finds an equation for the chemical potential Eq. (A1). See the Appendix for details.

We will use the Carnahan-Starling approximation Eq. (19) for $f^{0}$. For the hard sphere pair distribution function, we apply the Percus-Yevick approximation. Although more accurate descriptions of the hard sphere pair distribution function are available, e.g., through modifications of the fundamental measure theory, ${ }^{35,34}$ we believe that the PercusYevick framework is sufficient for our analysis, while having the advantage of an analytic solution. ${ }^{39,40}$

\section{THERMODYNAMIC VARIABLES}

In the further analysis, we need explicit expressions for the thermodynamic densities. Using the LDA it follows from Eq. (25) that

$$
\begin{aligned}
f(\mathbf{r})= & f^{0}(\rho(\mathbf{r}), T)+\frac{1}{2} \rho(\mathbf{r}) \int d \mathbf{r}^{\prime} g_{0}\left(\mathbf{r}, \mathbf{r}^{\prime}, \bar{\rho}\right) \rho\left(\mathbf{r}^{\prime}\right) u^{a}\left(\mathbf{r}, \mathbf{r}^{\prime}\right) \\
& -\frac{1}{4} \rho(\mathbf{r}) \int d \mathbf{r}^{\prime} \frac{\partial \bar{\rho}}{\partial p_{0}} g_{0}\left(\mathbf{r}, \mathbf{r}^{\prime}, \bar{\rho}\right) \rho\left(\mathbf{r}^{\prime}\right)\left[u^{a}\left(\mathbf{r}, \mathbf{r}^{\prime}\right)\right]^{2} .
\end{aligned}
$$

In view of the fact that the second order contribution gives not more then a $10 \%$ contribution, one may approximate the last term and use

$$
\begin{aligned}
f_{I I, A}(\mathbf{r})= & f(\rho(\mathbf{r}), T) \\
= & f^{0}(\rho(\mathbf{r}), T)+\frac{1}{2} \rho(\mathbf{r}) \int d \mathbf{r}^{\prime} g_{0}\left(\mathbf{r}, \mathbf{r}^{\prime}, \bar{\rho}\right) \rho\left(\mathbf{r}^{\prime}\right) u^{a}\left(\mathbf{r}, \mathbf{r}^{\prime}\right) \\
& -\frac{1}{4} \rho^{2}(\mathbf{r}) \frac{\partial \rho(\mathbf{r})}{\partial p_{0}} \int d \mathbf{r}^{\prime} g_{0}\left(\mathbf{r}, \mathbf{r}^{\prime}, \rho(\mathbf{r})\right)\left[u^{a}\left(\mathbf{r}, \mathbf{r}^{\prime}\right)\right]^{2} .
\end{aligned}
$$

Barker and Henderson argued that Eq. (27) should be improved, if not the macroscopic compressibility $\partial \bar{\rho} / \partial p_{0}$ was considered, but the local compressibility $\partial\left(\bar{\rho} g_{0}\left(\mathbf{r}, \mathbf{r}^{\prime}, \bar{\rho}\right)\right) / \partial p_{0}$. They proposed the following modification: ${ }^{31}$

$$
\begin{aligned}
f(\mathbf{r})= & f^{0}(\rho(\mathbf{r}), T)+\frac{1}{2} \rho(\mathbf{r}) \int d \mathbf{r}^{\prime} g_{0}\left(\mathbf{r}, \mathbf{r}^{\prime}, \bar{\rho}\right) \rho\left(\mathbf{r}^{\prime}\right) u^{a}\left(\mathbf{r}, \mathbf{r}^{\prime}\right) \\
& -\frac{1}{4} \rho(\mathbf{r}) \int d \mathbf{r}^{\prime} \frac{\partial \bar{\rho} g_{0}\left(\mathbf{r}, \mathbf{r}^{\prime}, \bar{\rho}\right)}{\partial p_{0}} \rho\left(\mathbf{r}^{\prime}\right)\left[u^{a}\left(\mathbf{r}, \mathbf{r}^{\prime}\right)\right]^{2} .
\end{aligned}
$$

Using again that the second order contribution gives not more then a $10 \%$ contribution, one may approximate the last term and use

$$
\begin{aligned}
f_{I I, B}(\mathbf{r})= & f^{0}(\rho(\mathbf{r}), T) \\
& +\frac{1}{2} \rho(\mathbf{r}) \int d \mathbf{r}^{\prime} g_{0}\left(\mathbf{r}, \mathbf{r}^{\prime}, \bar{\rho}\right) \rho\left(\mathbf{r}^{\prime}\right) u^{a}\left(\mathbf{r}, \mathbf{r}^{\prime}\right) \\
& -\frac{1}{4} \rho^{2}(\mathbf{r}) \int d \mathbf{r}^{\prime} \frac{\partial\left[\rho(\mathbf{r}) g_{0}\left(\mathbf{r}, \mathbf{r}^{\prime}, \rho(\mathbf{r})\right)\right]}{\partial p_{0}}\left[u^{a}\left(\mathbf{r}, \mathbf{r}^{\prime}\right)\right]^{2} .
\end{aligned}
$$

In both Eqs. (27) and (29), the last term can be calculated using the hard sphere results for all densities. The equilibrium density profile further follows in the usual way. In the further analysis, we will focus on the two approximations $f_{I I, A}$ and $f_{I I, B}$ given in Eqs. (27) and (29). Furthermore, we will for comparison also calculate the first order approximation, indicated by $f_{I}$. All thermodynamic quantities in this approximation are obtained from those given by neglecting the contribution quadratic in the attractive potential $u^{a}$.

The chemical potentials, the parallel pressures, and the entropy densities which correspond to the Helmholtz free energy densities in Eq. (27) and (29) are given in the Appendix. We furthermore define the internal energy density per particle as

$$
u(\mathbf{r}) \equiv \frac{f(\mathbf{r})}{\rho(\mathbf{r})}+T s(\mathbf{r})=T s(\mathbf{r})-p_{\|}(\mathbf{r}) v(\mathbf{r})+\mu
$$

and the molar enthalpy per particle by

$$
h(\mathbf{r}) \equiv u(\mathbf{r})+p_{\|}(\mathbf{r}) v(\mathbf{r})=T s(\mathbf{r})+\mu,
$$

where the volume per particle is $v=1 / \rho$. The definitions of $u_{I I, A}, u_{I I, B}$ and $h_{I I, A}, h_{I I, B}$ are the same with the appropriate subscripts. With the above definitions, $f, u, s, h, p_{\|}$, and $\mu$ are related by the thermodynamic relations given above. Other thermodynamic relations for $f, u, s, h, p_{\|}$, and $\mu$ are true, only when they can be derived from the above definitions. The same is true for $f_{I}, u_{I}, s_{I}, h_{I}, p_{\|, I}, \mu_{I} ; f_{I I, A}$, $u_{I I, A}, s_{I I, A}, h_{I I, A}, p_{\| I I I I, A}, \mu_{I I, A} \quad$ and $f_{I I, B}, u_{I I, B}, s_{I I, B}, h_{I I, B}$, $p_{\|, I I, B}, \mu_{I I, B}$. The parallel pressure, and not the normal pressure, satisfies the above thermodynamic relations. Sufficiently far from the surface the normal and the parallel pressure become identical and then also the normal pressure satisfies the above thermodynamic relations. We shall not give an explicit expression for the normal component of the pressure tensor. 
TABLE I. The intermolecular pair potential, LJ parameters for calculation of variables in reduced units, and references for the three cases studied.

\begin{tabular}{lllllc}
\hline \hline & Potential & \multicolumn{1}{c}{$r_{c} / \sigma$} & $\epsilon / k_{B}[\mathrm{~K}]$ & $\sigma[\AA]$ & Refs. \\
\hline Xu case & Spline & 1.73705 & 165 & 3.40 & 14,30 , and 12 \\
Ge case & Spline & 2.5 & 134 & 3.405 & 23 \\
Vrabec case & Shifted/truncated & 2.5 & 137.9 & 3.3916 & 28 \\
\hline \hline
\end{tabular}

\section{CALCULATIONS}

Calculations were done for the first order DFT and the two second order DFT's discussed in Secs. V and VI. For each DFT, bulk coexistence properties of the liquid and the vapor, profiles of density and thermodynamic variables through the equilibrium liquid-vapor interface, surface tension, and interfacial transfer coefficients are main results.

Three intermolecular pair potentials were studied; the LJ spline potential (see Eq. (15)) with cutoff $r_{c}=1.73705 \sigma$, the LJ-spline potential with cutoff $r_{c}=2.5 \sigma$, and the LJ-cutoff/ shifted potential (see Eq. (13)) with cutoff $r_{c}=2.5 \sigma$. When presenting the results, we shall refer to the different potentials as the $\mathrm{Xu}$ case, the Ge case, and the Vrabec-case, respectively. Details of the three cases are summarized in Table I. Xu et al. ${ }^{14}$ did NEMD simulations using the LJ-spline potential with cutoff $r_{c}=1.73705 \sigma$ and calculated interfacial transfer coefficients (see also the work by Røsjorde and co-workers ${ }^{30,12}$ ). Ge et al. ${ }^{23}$ did similar work for the LJspline potential with cutoff $r_{c}=2.5 \sigma$. And Vrabec et al. ${ }^{28}$ studied the liquid-vapor interface using DFT and the LJcutoff/shifted potential with cutoff $r_{c}=2.5 \sigma$.

For each combination of DFT and potential, the calculations for a given temperature were performed in the following way:

(1) The coexisting liquid and vapor densities, $\rho_{\mathrm{liq}}^{\mathrm{Cox}}(T)$ and $\rho_{\text {gas }}^{\text {Coex }}(T)$, were found as the solution of the standard phase equilibrium conditions; the chemical potential and the pressure are equal in both phases. In the calculation of chemical potentials and pressures, it was used that the density is constant in a bulk phase. Afterwards, bulk coexistence properties of the liquid and the vapor (Helmholtz free energy, internal energy, enthalpy, entropy, etc.) were calculated.

(2) The equilibrium density profiles were calculated from Eqs. (23), (A2), and (A3) for the first order DFT, the second order DFT A, and the second order DFT B, respectively. The chemical potential is constant through the equilibrium liquid-vapor interface. Profiles of thermodynamic properties through the interface were calculated from the equilibrium density profile.

(3) The positions $z^{s, l}$ and $z^{s, g}$, where the interfacial region begins and ends, were determined from the equilibrium density profile. In the interfacial region, the density changes from $\rho_{\mathrm{lig}}^{\mathrm{Coex}}(T)$ to $\rho_{\mathrm{gas}}^{\mathrm{Cox}}(T)$. We define $\kappa$ dependent positions $z^{s, l}(\kappa)$ and $z^{s, g}(\kappa)$ by

$$
\left|\frac{\rho\left(z^{s, l}(\kappa)\right)-\rho_{\text {loex }}^{\operatorname{Coex}}(T)}{\rho_{\text {liq }}^{\operatorname{Cox}}(T)}\right|=\kappa
$$

and $\left|\frac{\rho\left(z^{s, g}(\kappa)\right)-\rho_{\text {gas }}^{\text {Coex }}(T)}{\rho_{\text {gas }}^{\text {Cox }}(T)}\right|=\kappa$,

respectively. The parameter $\kappa$ is smaller than one and larger than zero. This means that the interfacial region is the part of the system where the local density deviates more than a factor $\kappa$ from both $\rho_{\text {liq }}^{\text {Coex }}(T)$ and $\rho_{\text {gas }}^{\text {Cox }}(T)$. The $\kappa$ dependent surface thickness is defined by

$D(\kappa)=\left|z_{\text {liq }}(\kappa)-z_{\text {gas }}(\kappa)\right|$.

(4) Extrapolated and excess densities were calculated as described by Johannessen and Bedeaux. ${ }^{19}$ We used the equimolar surface, which is defined by

$0=\int_{-z_{\text {end }}+1}^{z_{\text {end }}-1}\left[\rho(z)-\rho_{\text {liq }}(z) \theta\left(z^{s}-z\right)-\rho_{\text {gas }}(z) \theta\left(z-z^{s}\right)\right] d z$,

as the dividing surface throughout this study. Here, $\rho_{\text {liq }}(z)$ and $\rho_{\text {gas }}(z)$ are extrapolated densities on the liquid and on the gas sides, respectively, $\theta$ is the Heaviside function, and $z^{s}$ is the dividing surface.

The surface tension is the negative excess parallel pressure

$$
\begin{array}{r}
\gamma(T)=-\int_{-z_{\text {end }}}^{z_{\text {end }}}\left[p_{\|}(z)-p_{\|, \text {liq }}(z) \theta\left(z^{s}-z\right)\right. \\
\left.-p_{\|, \text {gas }}(z) \theta\left(z-z^{s}\right)\right] d z,
\end{array}
$$

where $p_{\|, \text {liq }}(z)$ and $p_{\|, \text {gas }}(z)$ are extrapolated parallel pressures on the liquid and on the gas sides, respectively.

Linear extrapolation was used in order to find extrapolated densities, because this is appropriate when the system is in global equilibrium. The extrapolated denstities are thus constant and equal to the corresponding bulk values. For instance, the extrapolated parallel pressures and densities are

$p_{\|, \text {liq }}(z)=p_{\|, \text {gas }}(z)=p^{\mathrm{Coex}}(T)$,

$\rho_{\text {liq }}(z)=\rho_{\text {liq }}^{\text {Coex }}(T)$,

$\rho_{\text {gas }}(z)=\rho_{\text {gas }}^{\text {Coex }}(T)$.

(5) Interfacial transfer coefficients were calculated with the integral relations, Eqs. (4) and (5), as described in Sec. I. There is no a priori way to estimate the overall size parameter $\alpha$ and the asymmetry parameter $\beta$, so for the $\mathrm{Xu}$ and $\mathrm{Ge}$ cases these constants where fitted to the 
TABLE II. Reduced units of variables.

\begin{tabular}{|c|c|}
\hline Distance & $\sigma$ \\
\hline Energy & $\epsilon$ \\
\hline Temperature & $\epsilon / k_{B}$ \\
\hline Pressure & $\epsilon / \sigma^{3}$ \\
\hline Density & $1 / \sigma^{3}$ \\
\hline Velocity & $\sqrt{\epsilon / m}$ \\
\hline de Broglie wavelength & $\sigma$ \\
\hline Entropy density & $k_{B}$ \\
\hline Internal energy density & $\epsilon$ \\
\hline Enthalpy density & $\epsilon$ \\
\hline Helmholtz free energy density & $\epsilon$ \\
\hline Chemical potential & $\epsilon$ \\
\hline Surface tension & $\epsilon / \sigma^{2}$ \\
\hline Thermal conductivity & $k_{B} / \sigma^{2} \sqrt{\epsilon / m}$ \\
\hline Mol flux & $1 / N_{A} \sigma^{3} \sqrt{\epsilon / m}$ \\
\hline Sensible heat and total heat/energy fluxes & $\boldsymbol{\epsilon} / \sigma^{3} \sqrt{\boldsymbol{\epsilon} / m}$ \\
\hline Overall size parameter ( $\alpha$ in Eq. (6)) & $\sigma^{10} / k_{B} \sqrt{m / \epsilon}$ \\
\hline \multicolumn{2}{|l|}{ Transfer coefficients } \\
\hline$R_{q q}^{g}, R_{q q}^{l}, R_{q q}^{s, g}, R_{q q}^{s, l}$ & $\left(\sigma^{3} / \epsilon^{2}\right) k_{B} \sqrt{m / \epsilon}$ \\
\hline$R_{q \mu}^{g}, R_{q \mu}^{l}, R_{q \mu}^{s, g}, R_{q \mu}^{s, l}, R_{\mu q}^{g}, R_{\mu q}^{l}, R_{\mu q}^{s, g}, R_{\mu q}^{s, l}$ & $N_{A}\left(\sigma^{3} / \epsilon\right) k_{B} \sqrt{m / \epsilon}$ \\
\hline$R_{\mu \mu}^{g}, R_{\mu \mu}^{l}, R_{\mu \mu}^{s, g}, R_{\mu \mu}^{s, l}$ & $N_{A}^{2} \sigma^{3} k_{B} \sqrt{m / \epsilon}$ \\
\hline
\end{tabular}

interfacial transfer coefficients that were calculated from NEMD simulation results. ${ }^{14,30,12,23}$ No interfacial transfer coefficients are available for the Vrabec case.

All results are given in reduced units, see Table II for definitions. The interfacial transfer coefficients calculated from NEMD simulation results are given in real units in the original papers. ${ }^{14,30,12,23}$ We back calculated the transfer coefficients to reduced units as described in Table II with the LJ parameters used in the original papers $\left(\sigma=3.42 \AA\right.$ and $\epsilon / k_{B}$ $=124 \mathrm{~K}$ ). Note that these values are different from the values of the LJ parameters given for the $\mathrm{Xu}$ and Ge cases in Table I. The LJ parameters in Table I are the values which give best fit between the phase diagram obtained with molecular dynamics (MD) and Monte Carlo (MC) simulations and the experimental phase diagram of argon, see Tegeler et al. ${ }^{41}$ for experimental data for argon. Vrabec et al. ${ }^{28}$ also used this approach to find $\sigma$ and $\epsilon / k_{B}$. In order to calculate interfacial transfer coefficients from the DFT/nonequilibrium thermodynamics description of the surface, the thermal conductivities of argon (liquid and vapor) along the coexistence curve ${ }^{42}$ were used for the bulk phases. The reduced values of the bulk thermal conductivities were calculated as described in Table II by using the LJ parameters in Table I.

\section{RESULTS AND DISCUSSION}

The results for the $\mathrm{Xu}, \mathrm{Ge}$, and Vrabec cases are qualitatively the same. Only results for the Ge case are presented here, if not stated otherwise.

\section{A. Phase equilibrium}

The phase diagram and the vapor pressure as a function of temperature are shown in Fig. 1. In both figures, lines are given for the first order DFT (dash-dotted lines), the second order DFT A (dashed lines), and the second order DFT B
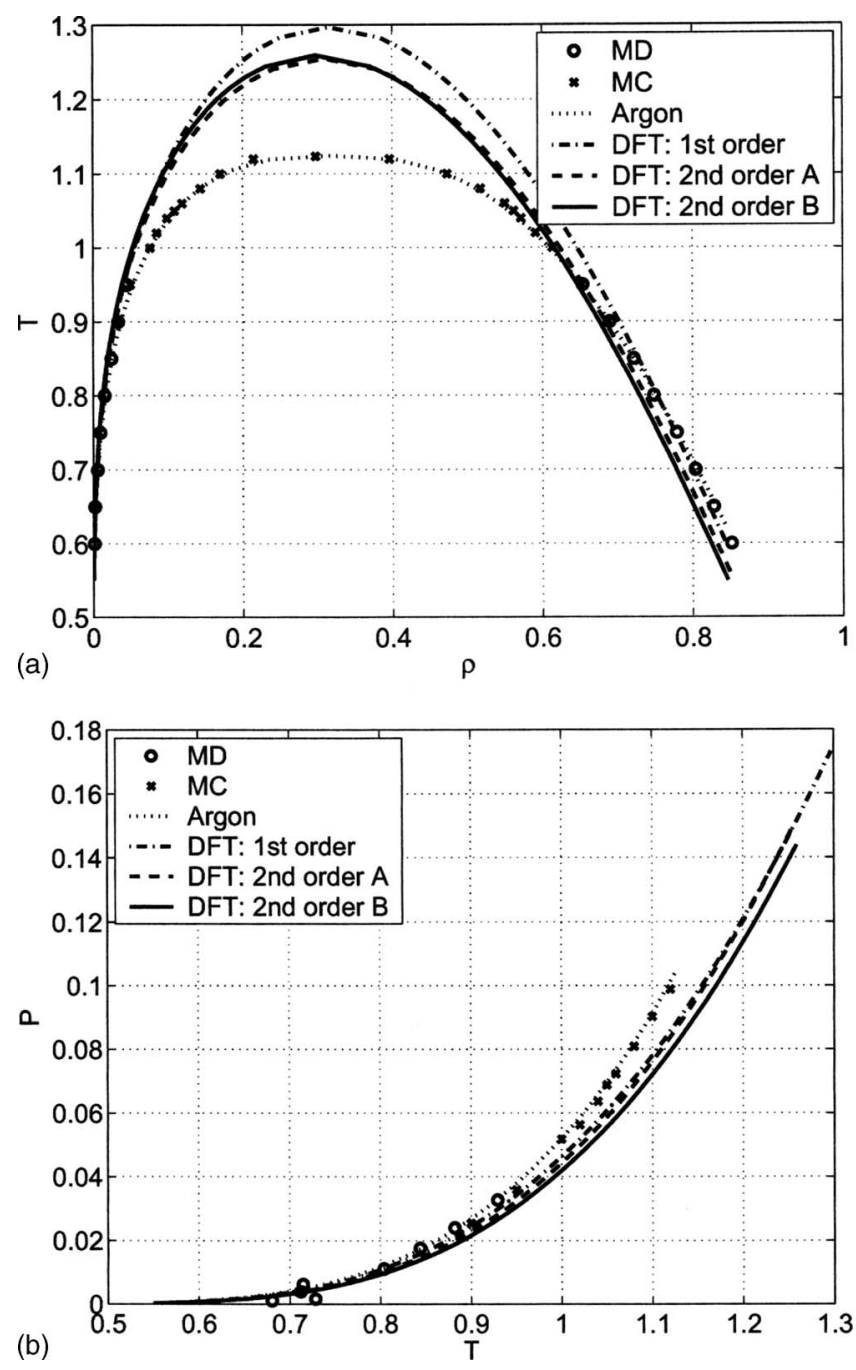

FIG. 1. Phase diagram (a) and vapor pressure vs temperature (b) for the Ge case (LJ spline with $r_{c}=2.5 \sigma$ ).

(solid lines). The experimental data for argon, ${ }^{41}$ presented in reduced units, are given with dotted lines. MD and MC data are given with points.

Figure 1 shows that the agreement between simulation and DFT results are good away from the critical region. The critical point is overestimated by all DFT's, but the critical point is predicted best by the second order DFT's. The deviations around the critical point are expected, since the long-wave fluctuations are not accounted for. A renormalization accounting for such fluctuations is not considered here.

For the phase diagram, part (a) of Fig. 1, the difference between the two second order theories is not significant. Away from the critical point, the second order DFT's underestimate the liquid density and the first order theory overestimates the liquid density. Furthermore, part (b) of Fig. 1 shows that the vapor pressure of the first order DFT and the second order DFT A fit better with simulation results than the vapor pressure of the second order DFT B.

The enthalpy of evaporation as a function of temperature is shown in Fig. 2. Ge et al. $^{23}$ neglected the temperature dependence and estimated the enthalpy of evaporation from vapor pressure versus temperature as found by MD and MC 


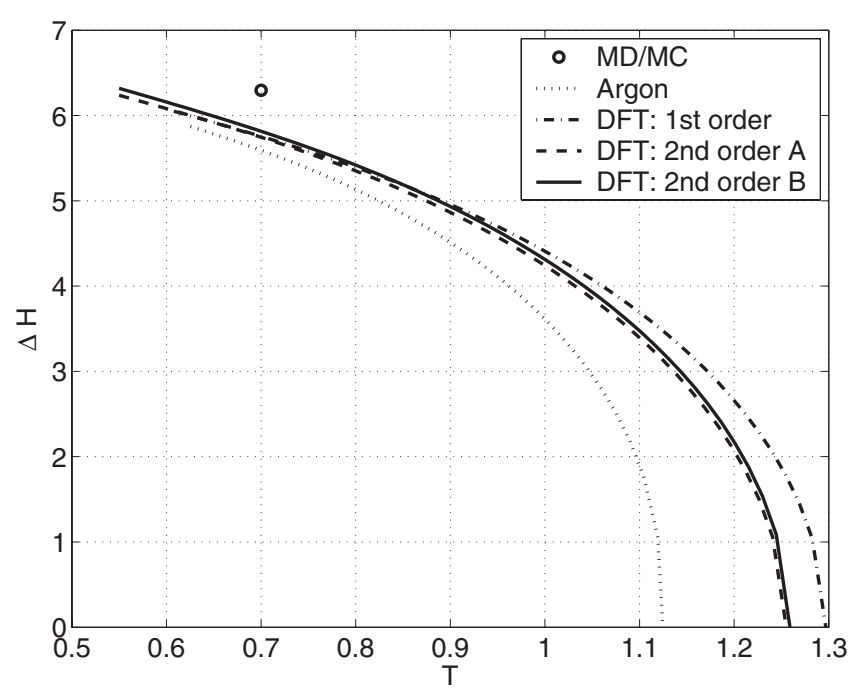

FIG. 2. Enthalpy of evaporation vs temperature for the Ge case (LJ spline with $r_{c}=2.5 \sigma$ ).

simulations, part (b) of Fig. 1. The enthalpy of evaporation that was estimated from simulation results is plotted at $T$ $=0.7$ in Fig. 2 .

Figure 2 shows again that the agreement between the DFT's and the MD (and MC) simulation results is good away from the critical point. Overall, the agreement between theory and simulations is best for the second order DFT's.

\section{B. Equilibrium profiles and surface tension}

Typical equilibrium density profiles for two different temperatures are shown in Fig. 3. The density decreases monotonically from the liquid to the vapor. The $\kappa$ dependent positions $z^{s, l}(\kappa)$ and $z^{s, g}(\kappa)$, see Sec. VII for the definition, are given for $\kappa=0.1,0.01,0.001$, and 0.0001 in Fig. 3. The interfacial region for $\kappa=0.1$ is the film in between the two central vertical dotted lines. The interfacial region for $\kappa$ $=0.01$ is given by the next set of vertical dotted lines (working outwards), and so on. As expected, the surface thickness for a given value of $\kappa$ increases when one approaches the critical point. In the NEMD simulations by Røsjorde and co-workers, ${ }^{30,12,14,15}$ the interfacial region is approximately the one for $\kappa=0.01$ in Fig. 3, so $\kappa=0.01$ will be used from now on.

Typical behavior of thermodynamic properties through the liquid-vapor interface is shown in Figs. 4-6. Profiles for the internal energy and the entropy are not displayed, but they show transitions similar to the enthalpy. The enthalpy is given in Fig. 4. We see that the value shifts from one value in the liquid to another value in the vapor. The vapor and liquid values are substantially different, and there is no special behavior in the interfacial region. The Helmholtz free energy, Fig. 5, and parallel pressure, Fig. 6, behave in a different manner. There are peaks in the profiles for these properties. The effect of temperature is illustrated by comparing parts (a) and (b) of Figs. 4-6. As the temperature increases, liquid and vapor properties approach each other, as expected, and the peaks in the interfacial region for the Helmholtz free energy and the parallel pressure become smaller.

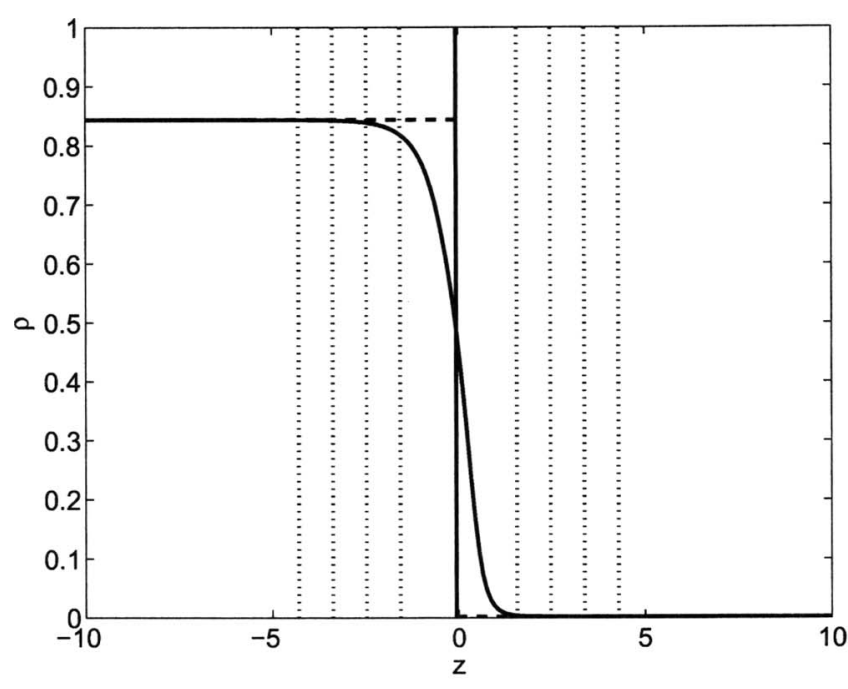

(a)

$$
T=0.60
$$

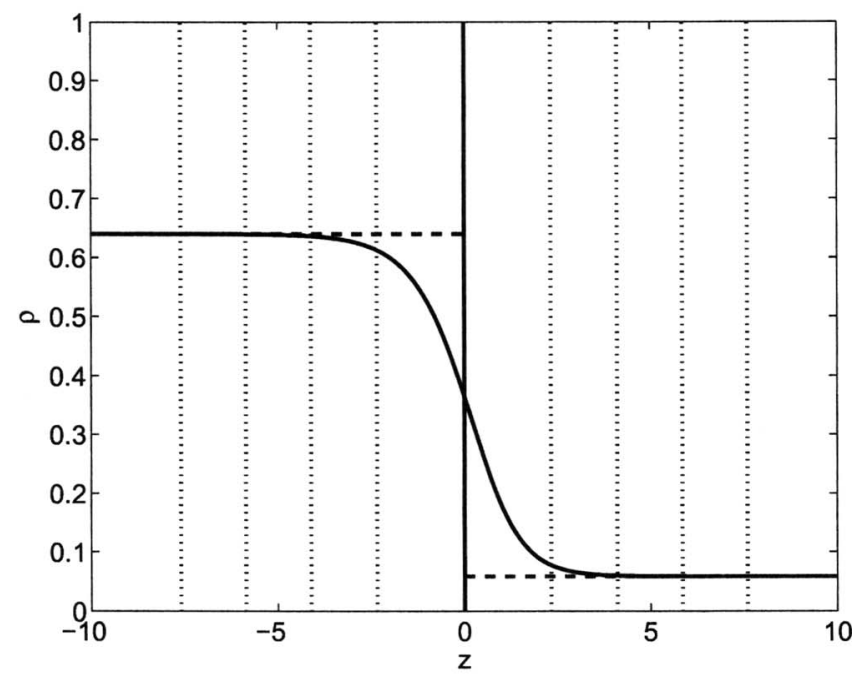

(b)

$$
T=1.01
$$

FIG. 3. Density profiles with the surface thickness indicated with vertical dashed lines. The dotted lines correspond to $\kappa=0.1,0.01,0.001$, and 0.0001 , with $\kappa=0.1$ given by the two lines closest to the equimolar surface (vertical solid line). These results are for the first order DFT and Ge case (LJ spline with $\left.r_{c}=2.5 \sigma\right)$.

As explained in Sec. VII, the surface tension is the negative excess parallel pressure, see Fig. 6. Figure 7 shows the surface tension versus temperature from the MD simulations and from the three DFT's. The surface tension of the second order theories agrees better with the simulations than the surface tension of the first order theory. This is consistent with the differences in critical temperatures.

\section{Interfacial transfer coefficients}

The calculation of the interfacial transfer coefficients for heat and mass transfer is described in Sec. VII. Figure 8 shows the effects of varying the overall size parameter $\alpha$ and the asymmetry parameter $\beta$ in the local heat transfer resistance, Eq. (6). In Figure 8(a), $\beta=3.1$ and $\alpha$ varies. The magnitude of the resistivity for heat transfer $R_{q q}^{g}=R_{q q}^{l}$ increases when $\alpha$ increases. In Fig. 8(b), $\alpha=1$ and $\beta$ varies. The magnitude of the coefficient increases when $\beta$ increases. Note 


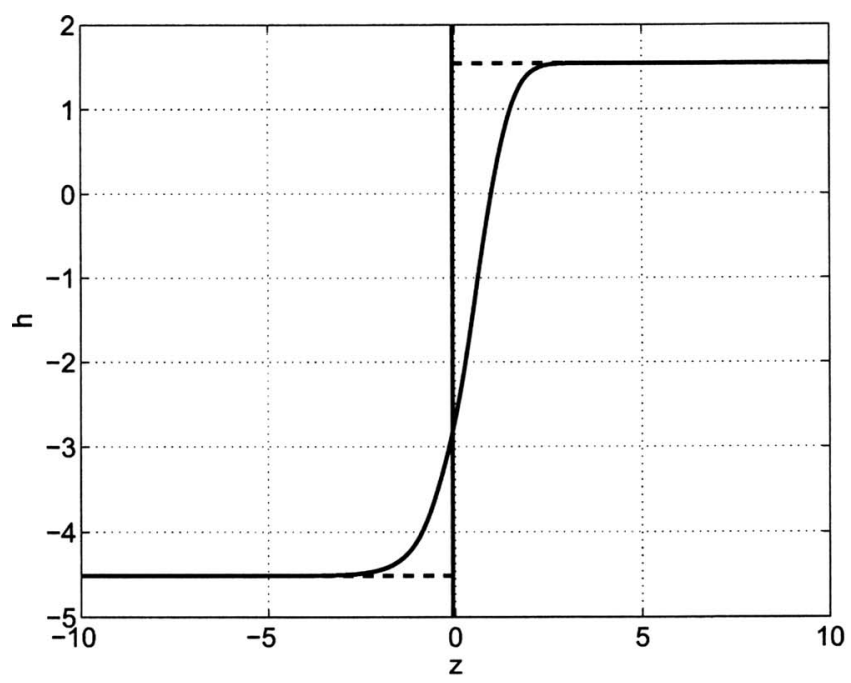

(a)

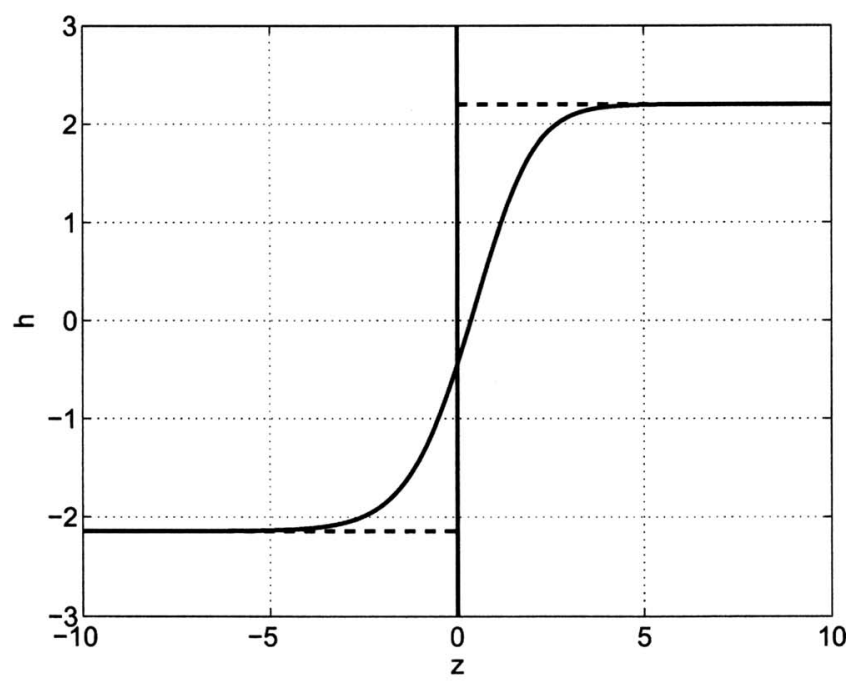

(b)

$T=1.01$

FIG. 4. The enthalpy density. These results are for the first order DFT and Ge case (LJ spline with $r_{c}=2.5 \sigma$ ).

that $\alpha$ has a dimension in Eq. (6) and that $\alpha$ is given in reduced units here. Qualitatively, the behavior of the other interfacial transfer resistivities both on the vapor and the liquid sides of the surface is the same. When one approaches the critical point the local resistivity for heat transfer $r_{q q}$ and the enthalpy density $h$ per unit of volume in the liquid and the vapor approach each other. We then find that the value of $R_{q q}^{g}=R_{q q}^{l}$ becomes small and equal to the value of $r_{q q}$ in the critical point times the thickness of the surface, $z^{s, g}-z^{s, l}$, while the other elements in the resistivity matrix approach zero.

In order to compare the values of the interfacial resistivities for heat and mass transfer to those obtained in the NEMD simulations, we fitted the values of $\alpha$ and $\beta$ to the NEMD results. The values one obtains for $\alpha$ and $\beta$ depend on the DFT chosen, but the agreement between simulation results and DFT results is equally good for all DFT's.

In Figs. 9-11, interfacial transfer coefficients obtained from NEMD simulations for the $\mathrm{Xu}$ case and the Ge case are given with points. The lines give the interfacial transfer co-

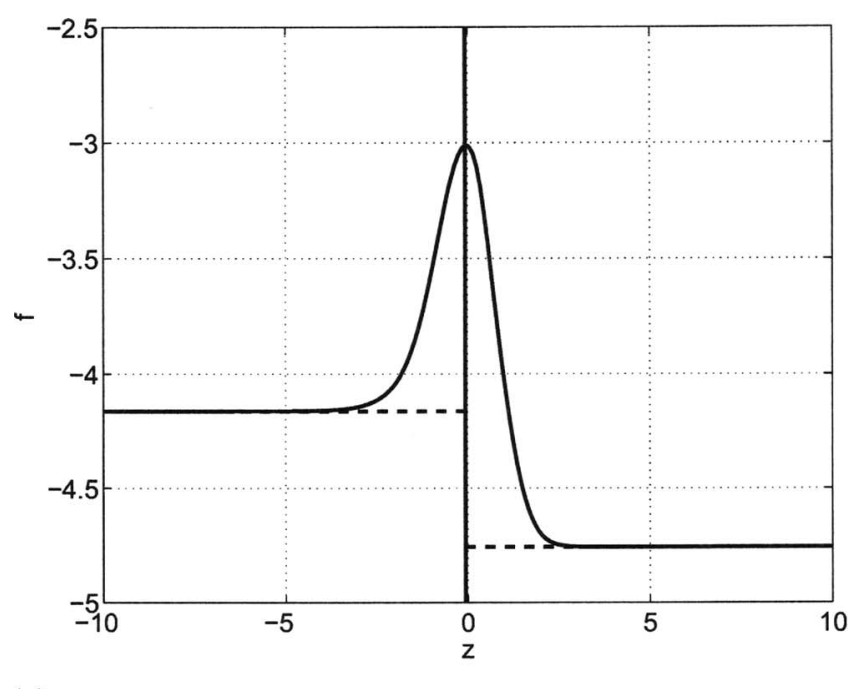

(a)

$T=0.60$

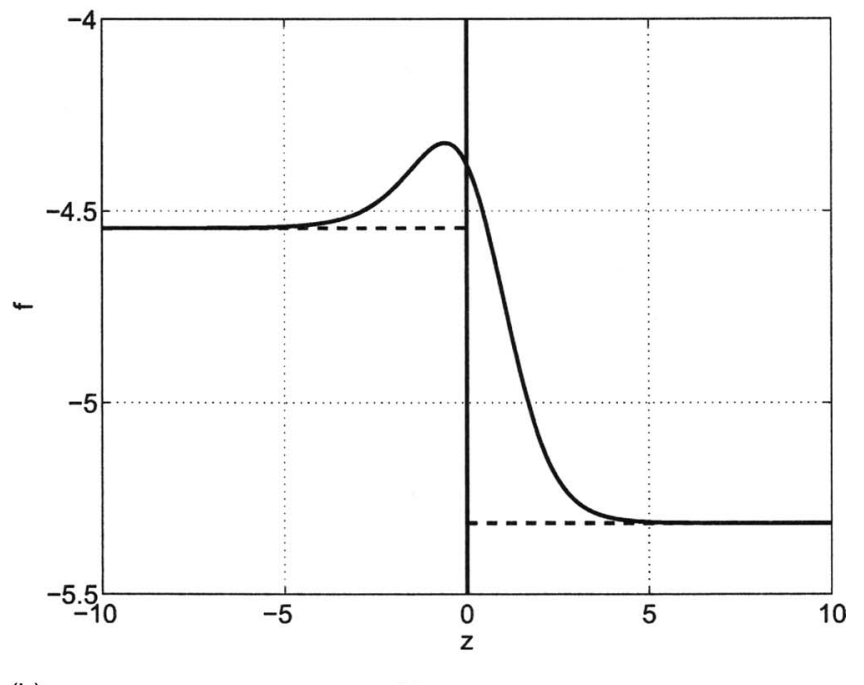

(b)

$T=1.01$

FIG. 5. The Helmholtz free energy density. These results are for the first order DFT and Ge case (LJ spline with $r_{c}=2.5 \sigma$ ).

efficients calculated from the second order DFT B. The lines for the Xu case (dash-dotted lines) are for $\alpha=1.66$ and $\beta$ $=2.86$, whereas the lines for the Ge case (solid lines) and the Vrabec case (dashed lines) are for $\alpha=0.65$ and $\beta=3.12$. The agreement between NEMD results and the DFT results is good, especially for $R_{q q}^{l}=R_{q q}^{g}$ and for the liquid side coefficients (see Figs. 9 and 10). The agreement with the gas side coefficients (see Fig. 11) is worse, but the relative uncertainty of the NEMD values is much larger for these coefficients because the absolute values are generally small. ${ }^{14,30,12}$

The values of the overall size parameter $\alpha$ and the asymmetry parameter $\beta$ that give best fit between NEMD results and model predictions depend on the DFT used. As discussed above, $\alpha=0.65$ and $\beta=3.12$ for the Ge case when the second order DFT B is used. When the first order DFT is used, best fit between NEMD results and model predictions was obtained with $\alpha=0.96$ and $\beta=3.06$ for this potential. Similary, for the $\mathrm{Xu}$ case best fit was obtained with $\alpha=1.66$ and $\beta$ $=2.86$ for the second order DFT B, whereas $\alpha=2.43$ and $\beta$ $=2.81$ gave best fit for the first order DFT. 


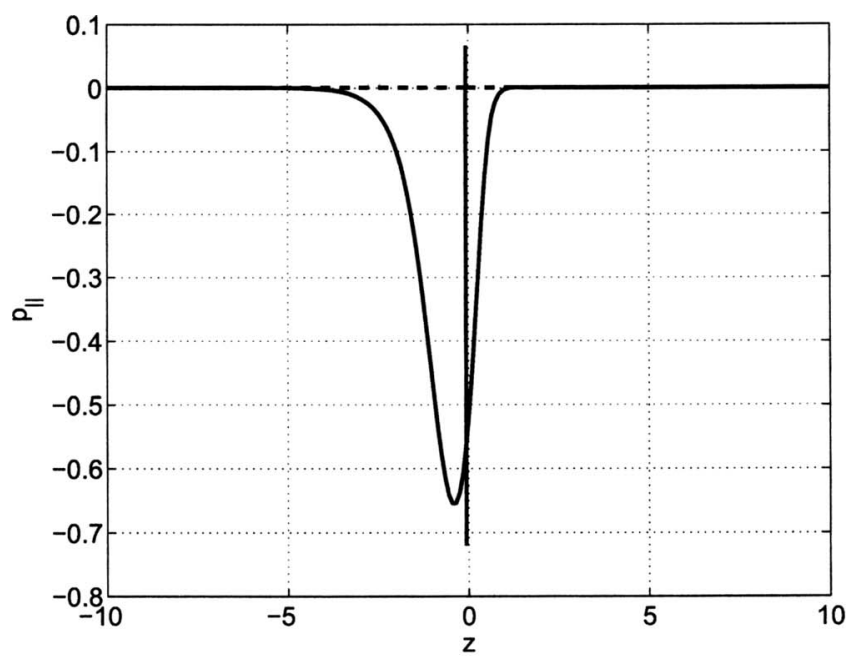

(a)

$$
T=0.60
$$

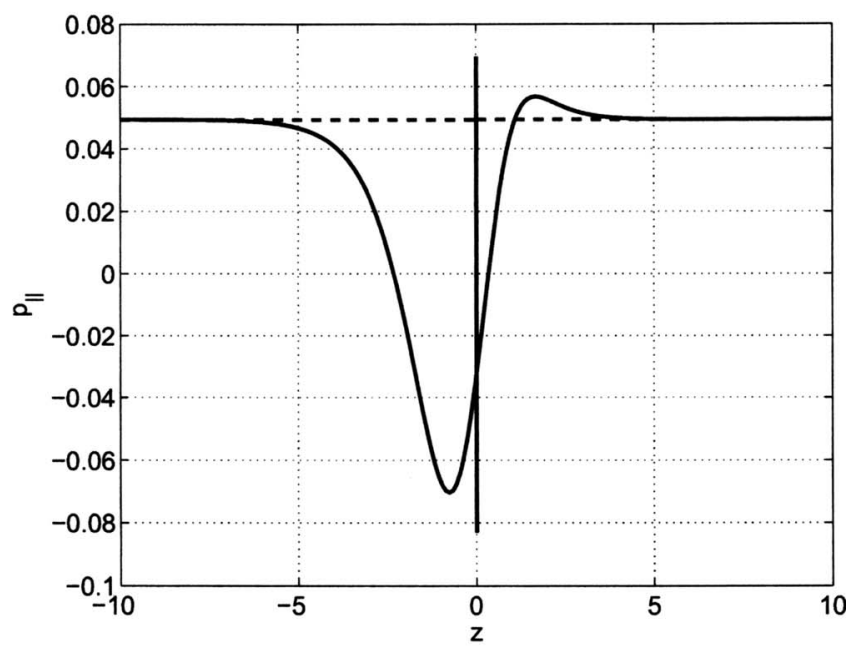

(b)

$$
T=1.01
$$

FIG. 6. The parallel pressure. These results are for the first order DFT and Ge case (LJ spline with $r_{c}=2.5 \sigma$ ).

An important result is that both the asymmetry parameter $\beta$ and the overall size parameter $\alpha$ are found to be independent of the local temperature and density. These constant values are appropriate to predict the complete temperature dependence of the resistivity matrices. The functional form for the third contribution to $r_{q q}$ given in Eq. (6) is therefore very appropriate. Other functional forms containing a square gradient term were tried but were found to be less satisfactory. Given the good agreement of the predictions with the NEMD data, there was no reason to search for a form with a different functional dependence on the first and the second order gradients.

\section{CONCLUDING REMARKS}

We conclude that the integral relations for the interfacial resistivities of heat and mass transfer through the surface can be combined with the results of density functional theories to obtain realistic values for the interfacial transfer coefficients. The analysis uses Eq. (6) for the local heat transfer resistance. This expression is the sum of three terms. The first two are an interpolation of tabulated values of the resistances at

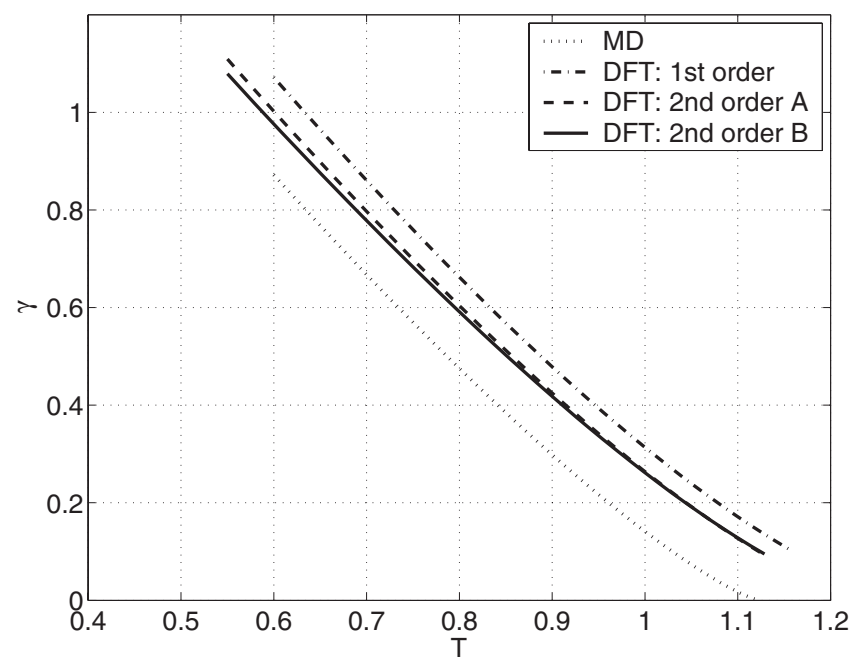

FIG. 7. Surface tension vs temperature. These results are for the Ge case (LJ spline with $\left.r_{c}=2.5 \sigma\right)$.

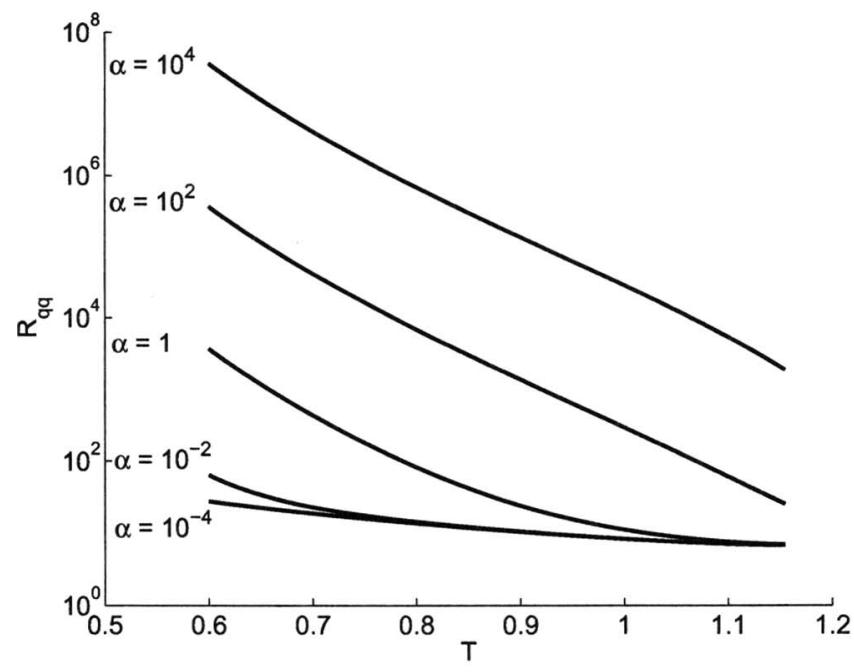

(a)

$\beta=3.1$

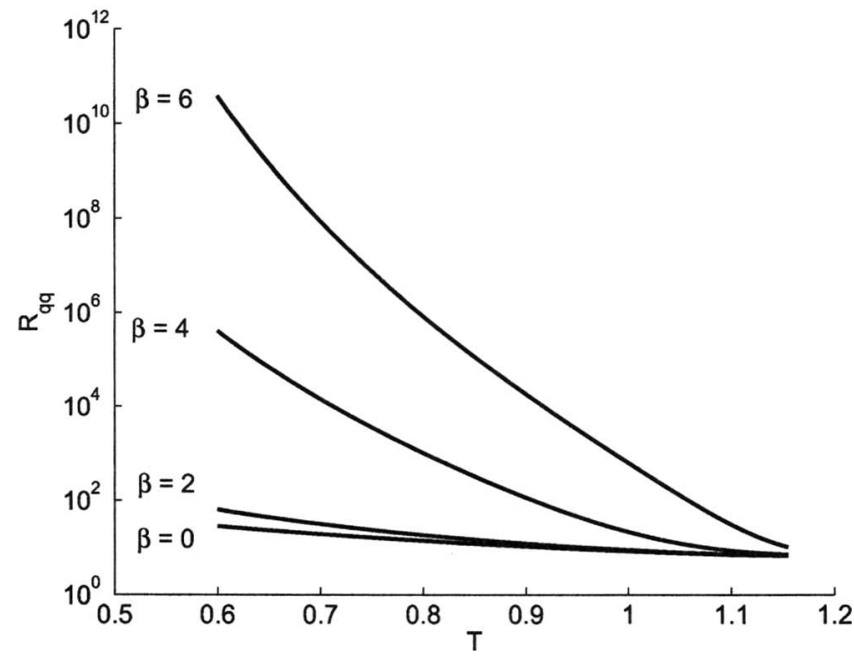

(b)

$\alpha=1$

FIG. 8. The effects of $\alpha$ and $\beta$ on the transfer coefficients. These results are for the first order DFT and Ge case (LJ spline with $r_{c}=2.5 \sigma$ ). 


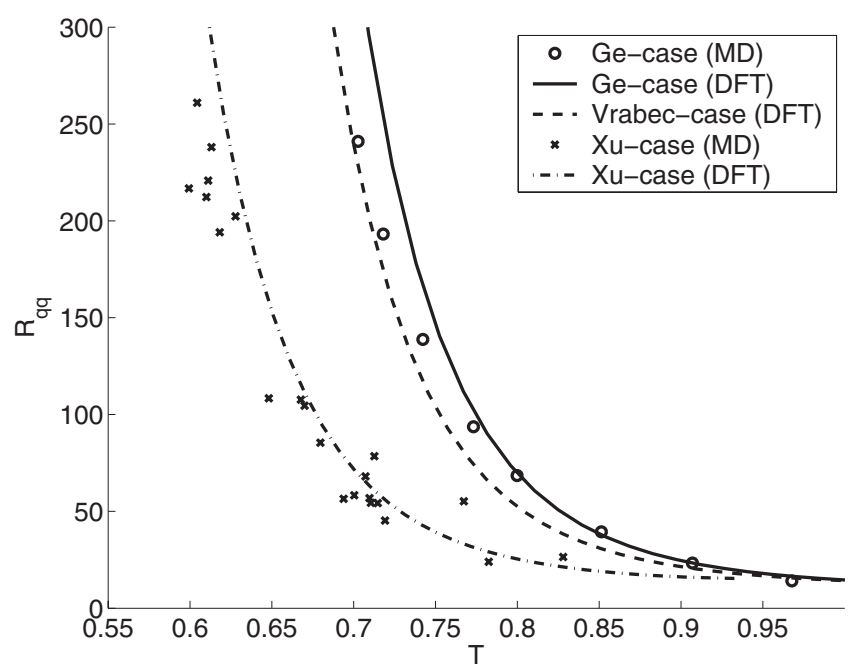

FIG. 9. $R_{q q}^{l}=R_{q q}^{g}$ calculated from MD results and from DFT results for the Ge case, Vrabec case, and the Xu case. The DFT results were obtained with the second order DFT B with $\alpha=0.65$ and $\beta=3.12$ for the Ge and Vrabec cases, and $\alpha=1.66$, and $\beta=2.86$ for the $\mathrm{Xu}$ case.

(a)
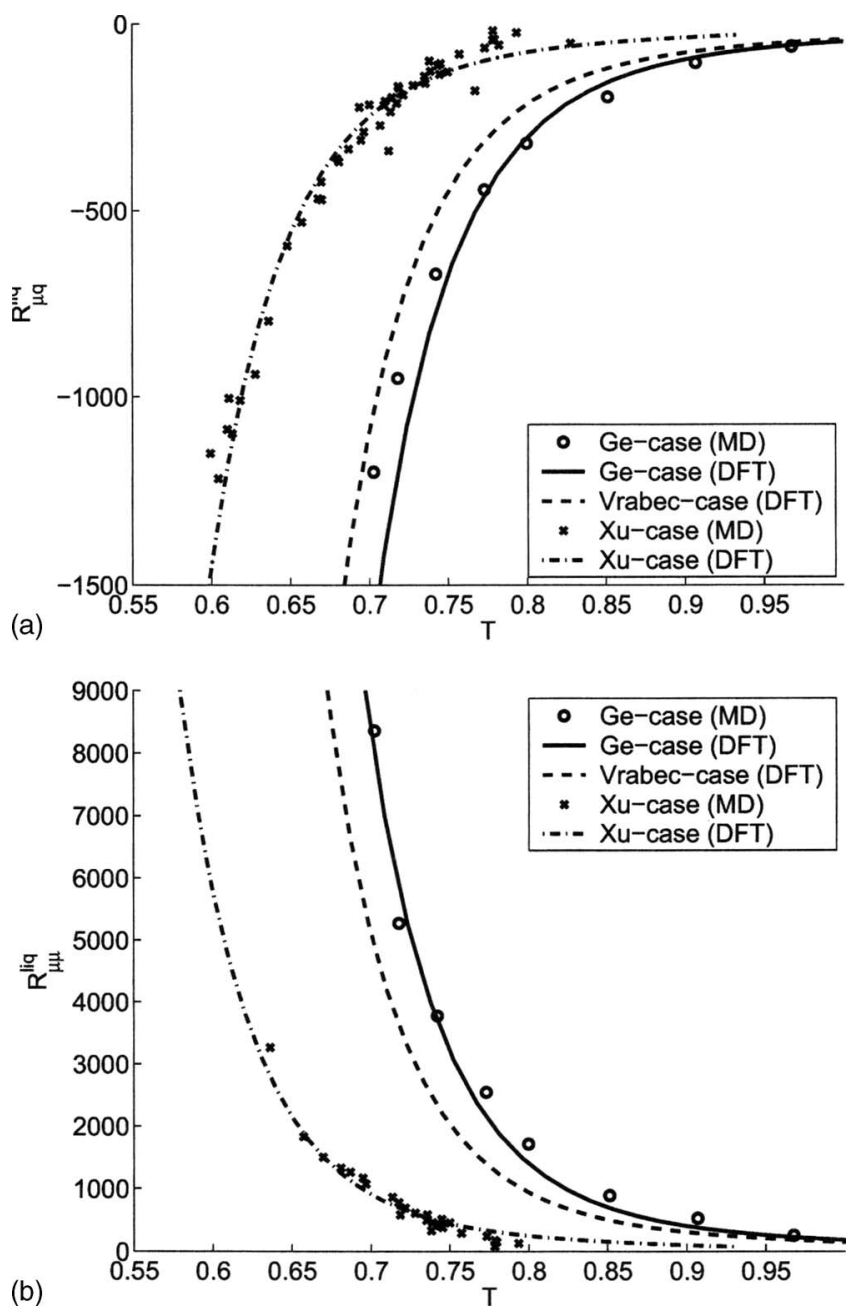

FIG. 10. $R_{\mu q}^{l}$ and $R_{\mu \mu}^{l}$ calculated from MD results and from DFT results for the Ge case, Vrabec case, and the Xu case. The DFT results were obtained with the second order DFT B with $\alpha=0.65$ and $\beta=3.12$ for the Ge and Vrabec cases, and $\alpha=1.66$ and $\beta=2.86$ for the Xu case.
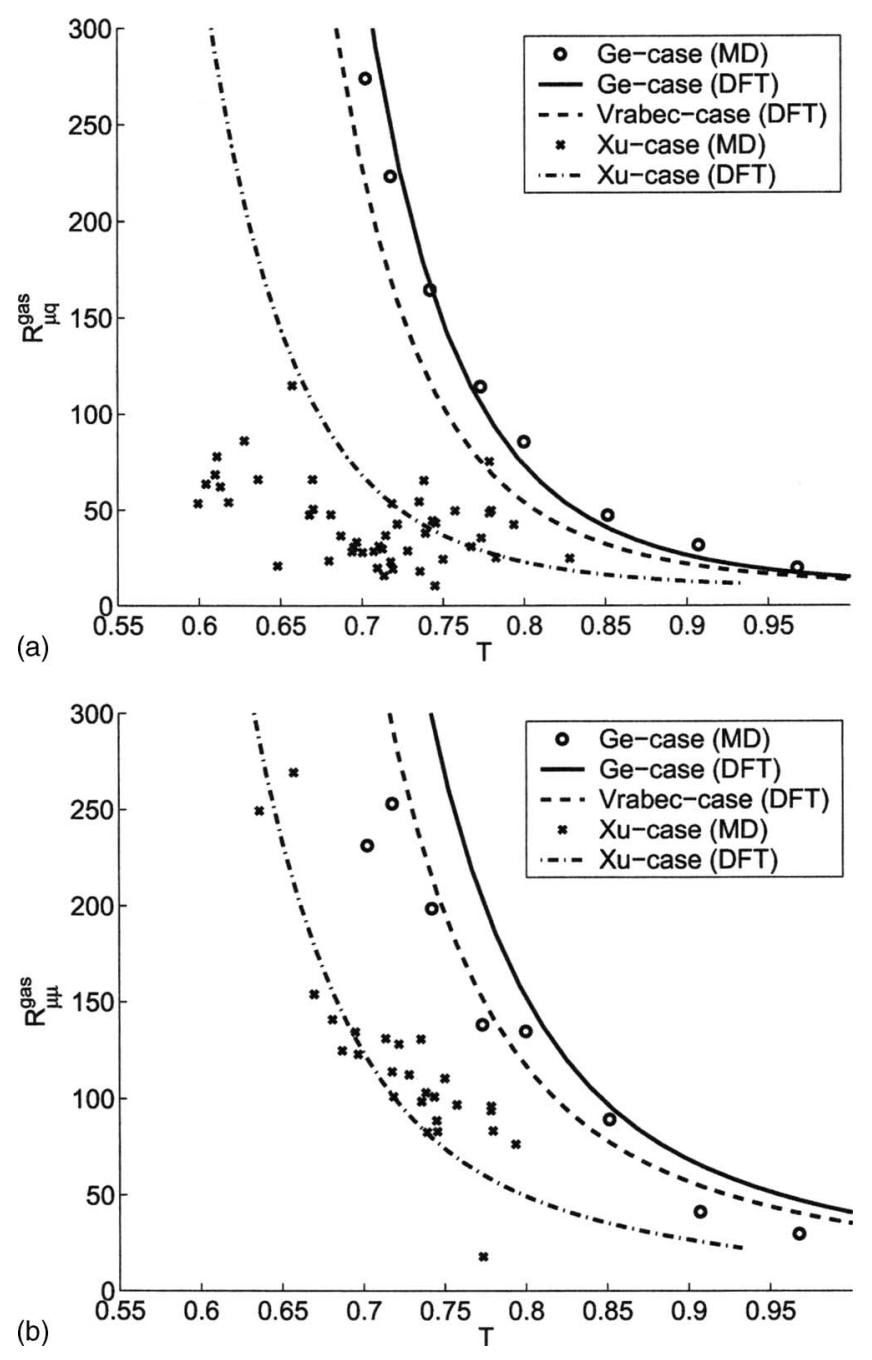

FIG. 11. $R_{\mu q}^{g}$ and $R_{\mu \mu}^{g}$ calculated from MD results and from DFT results for the Ge case, Vrabec case, and the Xu case. The DFT results were obtained with the second order DFT B with $\alpha=0.65$ and $\beta=3.12$ for the Ge and Vrabec cases, and $\alpha=1.66$ and $\beta=2.86$ for the Xu case.

coexistance in the homogeneous phases. The third term gives a contribution specific for the interfacial region proportional to the square gradient of the density. As was discussed in Sec. VIII C, a prediction of the resistivity matrices, which agreed well with the results of the NEMD simulations, was found for all temperatures between the triple and the critical points, using an asymmetry parameter $\beta$ and an overall size parameter $\alpha$ which were both independent of the local density and temperature. We conclude that the functional form of the local resistivity for heat transfer $r_{q q}$, and in particular of the third contribution due to the interface, is satisfactory. For the asymmetry parameter $\beta$, we found 3.12 (3.06) for the LJ potential with a longer range cutoff and 2.86 (2.81) for the shorter range cutoff when the second (first) order DFT was used. The overall size parameter $\alpha$ was 0.65 (0.96) for the longer range cutoff and 1.66 (2.43) for the shorter range cutoff when the second (first) order DFT was used. Clearly $\beta$ depends weakly, while $\alpha$ depends more strongly, on the range of the potential and the DFT used.

Other methods used to obtain the resistivity matrices for the surface are experiments, ${ }^{2,16,17}$ kinetic theory ${ }^{8-12,5}$ and NEMD simulations. ${ }^{12-15}$ Experiments lead to values that are 
larger than those found using the other two methods. One of the reasons is that it is difficult to avoid Marangoni convection along the surface. ${ }^{43}$ For the short range LJ-spline potential the predictions by kinetic theory and NEMD simulations agree with each other. ${ }^{12,14}$ For longer range potentials the resistivity coefficients, in particular the coupling coefficients, increase compared to the prediction from kinetic theory. ${ }^{14}$

The method presented in this paper has the great advantage that it is computationally cheap. One only needs equilibrium properties. The use of DFT to obtain the equilibrium properties of the system makes the analysis considerably more realistic than the one given earlier using the square gradient theory. ${ }^{18-20}$ The NEMD simulations are much more time consuming and, as this paper shows, give essentially the same results. In general it seems reasonable to assume that $\beta$ is about 3 while $\alpha$ varies between 0.5 and 2.5 depending on the range of the potential and the DFT used. This will make a calculation of the resistivity matrices for a given potential rather straightforward.

\section{ACKNOWLEDGMENTS}

E.J. and D.B. would like to thank the Norwegian Research Council for the grant "Transport at the nanoscale, surfaces, and contact lines."

\section{APPENDIX: THERMODYNAMIC VARIABLES}

Substituting Eq. (25) into Eq. (9) one finds, using the symmetry of $g_{0}\left(\mathbf{r}_{1}, \mathbf{r}_{2} ; \bar{\rho}_{12}\right)$ for the interchange of $\mathbf{r}_{1}$ and $\mathbf{r}_{2}$, the following equation for the chemical potential:

$$
\begin{aligned}
\mu= & \frac{\partial f^{0}\left(\rho_{\mathrm{eq}}(\mathbf{r}), T\right)}{\partial \rho_{\mathrm{eq}}(\mathbf{r})}+\int d \mathbf{r}^{\prime}\left[g_{0}\left(\mathbf{r}, \mathbf{r}^{\prime} ; \bar{\rho}\right)+\rho_{\mathrm{eq}}(\mathbf{r}) \frac{\partial g_{0}\left(\mathbf{r}, \mathbf{r}^{\prime} ; \bar{\rho}\right)}{\partial \rho_{\mathrm{eq}}(\mathbf{r})}\right] \rho_{\mathrm{eq}}\left(\mathbf{r}^{\prime}\right) u^{a}\left(\mathbf{r}, \mathbf{r}^{\prime}\right) \\
& -\left.\frac{1}{2} \int d \mathbf{r}^{\prime} \frac{\partial \bar{\rho}}{\partial p_{0}}\right|_{\mathrm{eq}}\left[g_{0}\left(\mathbf{r}, \mathbf{r}^{\prime} ; \bar{\rho}\right)+\rho_{\mathrm{eq}}(\mathbf{r}) \frac{\partial g_{0}\left(\mathbf{r}, \mathbf{r}^{\prime} ; \bar{\rho}\right)}{\partial \rho_{\mathrm{eq}}(\mathbf{r})}\right] \rho_{\mathrm{eq}}\left(\mathbf{r}^{\prime}\right)\left[u^{a}\left(\mathbf{r}, \mathbf{r}^{\prime}\right)\right]^{2} \\
& -\frac{1}{4}\left[\frac{\partial}{\partial \rho(\mathbf{r})}\left(\frac{\partial \rho(\mathbf{r})}{\partial p_{0}}\right)\right]_{\mathrm{eq}} \rho_{\mathrm{eq}}(\mathbf{r}) \int d \mathbf{r}^{\prime} g_{0}\left(\mathbf{r}, \mathbf{r}^{\prime} ; \bar{\rho}\right) \rho_{\mathrm{eq}}\left(\mathbf{r}^{\prime}\right)\left[u^{a}\left(\mathbf{r}, \mathbf{r}^{\prime}\right)\right]^{2} \\
= & \frac{\partial f^{0}\left(\rho_{\mathrm{eq}}(\mathbf{r}), T\right)}{\partial \rho_{\mathrm{eq}}(\mathbf{r})}+\int d \mathbf{r}^{\prime}\left[g_{0}\left(\mathbf{r}, \mathbf{r}^{\prime} ; \bar{\rho}\right)+\frac{1}{2} \rho_{\mathrm{eq}}(\mathbf{r}) \frac{\partial g_{0}\left(\mathbf{r}, \mathbf{r}^{\prime} ; \bar{\rho}\right)}{\partial \bar{\rho}}\right] \rho_{\mathrm{eq}}\left(\mathbf{r}^{\prime}\right) u^{a}\left(\mathbf{r}, \mathbf{r}^{\prime}\right)-\left.\frac{1}{2} \int d \mathbf{r}^{\prime} \frac{\partial \bar{\rho}}{\partial p_{0}}\right|_{\mathrm{eq}}\left[g_{0}\left(\mathbf{r}, \mathbf{r}^{\prime} ; \bar{\rho}\right)\right. \\
& \left.+\frac{1}{2} \rho_{\mathrm{eq}}(\mathbf{r}) \frac{\partial g_{0}\left(\mathbf{r}, \mathbf{r}^{\prime} ; \bar{\rho}\right)}{\partial \bar{\rho}}\right] \rho_{\mathrm{eq}}\left(\mathbf{r}^{\prime}\right)\left[u^{a}\left(\mathbf{r}, \mathbf{r}^{\prime}\right)\right]^{2}-\frac{1}{4}\left[\frac{\partial}{\partial \rho(\mathbf{r})}\left(\frac{\partial \rho(\mathbf{r})}{\partial p_{0}}\right)\right]_{\mathrm{eq}} \rho_{\mathrm{eq}}(\mathbf{r}) \int d \mathbf{r}^{\prime} g_{0}\left(\mathbf{r}, \mathbf{r}^{\prime} ; \bar{\rho}\right) \rho_{\mathrm{eq}}\left(\mathbf{r}^{\prime}\right)\left[u^{a}\left(\mathbf{r}, \mathbf{r}^{\prime}\right)\right]^{2},
\end{aligned}
$$

where $\bar{\rho}=1 / 2\left[\rho(\mathbf{r})+\rho\left(\mathbf{r}^{\prime}\right)\right]$.

Using the approximations that gave Eq. (27) for the Helmholtz energy, Eq. (A1) gives for the chemical potential

$$
\begin{aligned}
\mu_{I I, A}= & \frac{\partial f^{0}(\rho(\mathbf{r}), T)}{\partial \rho(\mathbf{r})}+\int d \mathbf{r}^{\prime}\left[g_{0}\left(\mathbf{r}, \mathbf{r}^{\prime} ; \bar{\rho}\right)\right. \\
& \left.+\frac{1}{2} \rho(\mathbf{r}) \frac{\partial g_{0}\left(\mathbf{r}, \mathbf{r}^{\prime}, \bar{\rho}\right)}{\partial \bar{\rho}}\right] \rho\left(\mathbf{r}^{\prime}\right) u^{a}\left(\mathbf{r}, \mathbf{r}^{\prime}\right) \\
& -\frac{1}{4} \frac{\partial}{\partial \rho(\mathbf{r})}\left[\rho^{2}(\mathbf{r}) \frac{\partial \rho(\mathbf{r})}{\partial p_{0}} \int d \mathbf{r}^{\prime} g_{0}\left[\mathbf{r}, \mathbf{r}^{\prime}, \rho(\mathbf{r})\right]\right. \\
& \left.\times\left[u^{a}\left(\mathbf{r}, \mathbf{r}^{\prime}\right)\right]^{2}\right] .
\end{aligned}
$$

Using the other approximation which gave Eq. (29) for the Helmholtz energy, one obtains

$$
\begin{aligned}
\mu_{I I, B}= & \frac{\partial f^{0}(\rho(\mathbf{r}), T)}{\partial \rho(\mathbf{r})}+\int d \mathbf{r}^{\prime}\left[g_{0}\left(\mathbf{r}, \mathbf{r}^{\prime}, \bar{\rho}\right)\right. \\
& \left.+\frac{1}{2} \rho(\mathbf{r}) \frac{\partial g_{0}\left(\mathbf{r}, \mathbf{r}^{\prime}, \bar{\rho}\right)}{\partial \bar{\rho}}\right] \rho\left(\mathbf{r}^{\prime}\right) u^{a}\left(\mathbf{r}, \mathbf{r}^{\prime}\right) \\
& -\frac{1}{4} \frac{\partial}{\partial \rho(\mathbf{r})}\left[\rho^{2}(\mathbf{r}) \int d \mathbf{r}^{\prime} \frac{\partial\left\{\rho(\mathbf{r}) g_{0}\left[\mathbf{r}, \mathbf{r}^{\prime}, \rho(\mathbf{r})\right]\right\}}{\partial p_{0}}\right. \\
& \left.\times\left[u^{a}\left(\mathbf{r}, \mathbf{r}^{\prime}\right)\right]^{2}\right] .
\end{aligned}
$$

For the pressure parallel to the surface, we may use

$$
\begin{aligned}
p_{\|}(\mathbf{r})= & \rho(\mathbf{r}) \mu-f(\mathbf{r}) \\
= & p^{0}(\mathbf{r})+\frac{1}{2} \rho(\mathbf{r}) \int d \mathbf{r}^{\prime}\left[g_{0}\left(\mathbf{r}, \mathbf{r}^{\prime}, \rho\right)\right. \\
& \left.+\rho(\mathbf{r}) \frac{\partial g_{0}\left(\mathbf{r}, \mathbf{r}^{\prime}, \bar{\rho}\right)}{\partial \bar{\rho}}\right] \rho\left(\mathbf{r}^{\prime}\right) u^{a}\left(\mathbf{r}, \mathbf{r}^{\prime}\right)
\end{aligned}
$$




$$
\begin{aligned}
& -\frac{1}{4} \rho(\mathbf{r}) \int d \mathbf{r}^{\prime} \frac{\partial \bar{\rho}}{\partial p_{0}}\left[g_{0}\left(\mathbf{r}, \mathbf{r}^{\prime}, \bar{\rho}\right)\right. \\
& \left.+\rho(\mathbf{r}) \frac{\partial g_{0}\left(\mathbf{r}, \mathbf{r}^{\prime}, \bar{\rho}\right)}{\partial \bar{\rho}}\right] \rho\left(\mathbf{r}^{\prime}\right)\left[u^{a}\left(\mathbf{r}, \mathbf{r}^{\prime}\right)\right]^{2} \\
& -\frac{1}{4} \rho(\mathbf{r}) \frac{\partial}{\partial \rho(\mathbf{r})}\left(\frac{\partial \rho(\mathbf{r})}{\partial p_{0}}\right) \int d \mathbf{r}^{\prime} g_{0}\left(\mathbf{r}, \mathbf{r}^{\prime}, \bar{\rho}\right) \rho(\mathbf{r}) \rho\left(\mathbf{r}^{\prime}\right) \\
& \times\left[u^{a}\left(\mathbf{r}, \mathbf{r}^{\prime}\right)\right]^{2},
\end{aligned}
$$

where we used Eq. (26), which gives the most symmetric expression. Using the approximation that gave Eq. (27) for the Helmholtz energy, one obtains

$$
\begin{aligned}
p_{\|, I I, A}(\mathbf{r})= & \rho(\mathbf{r}) \mu_{I I, A}-f_{I I, A}(\mathbf{r}) \\
= & p^{0}(\mathbf{r})+\frac{1}{2} \rho(\mathbf{r}) \int d \mathbf{r}^{\prime}\left[g_{0}\left(\mathbf{r}, \mathbf{r}^{\prime}, \bar{\rho}\right)\right. \\
& \left.+\rho(\mathbf{r}) \frac{\partial g_{0}\left(\mathbf{r}, \mathbf{r}^{\prime}, \bar{\rho}\right)}{\partial \bar{\rho}}\right] \rho\left(\mathbf{r}^{\prime}\right) u^{a}\left(\mathbf{r}, \mathbf{r}^{\prime}\right) \\
& -\frac{1}{4} \rho^{2}(\mathbf{r}) \frac{\partial}{\partial \rho(\mathbf{r})}\left[\rho(\mathbf{r}) \frac{\partial \rho(\mathbf{r})}{\partial p_{0}} \int d \mathbf{r}^{\prime} g_{0}\left(\mathbf{r}, \mathbf{r}^{\prime}, \rho(\mathbf{r})\right)\right. \\
& \left.\times\left[u^{a}\left(\mathbf{r}, \mathbf{r}^{\prime}\right)\right]^{2}\right] .
\end{aligned}
$$

Using the approximation that gave Eq. (29) for the Helmholtz energy, one obtains

$$
\begin{aligned}
p_{\|, I I, B}(\mathbf{r})= & \rho(\mathbf{r}) \mu_{I I, B}-f_{I I, B}(\mathbf{r}) \\
= & p^{0}(\mathbf{r})+\frac{1}{2} \rho(\mathbf{r}) \int d \mathbf{r}^{\prime}\left[g_{0}\left(\mathbf{r}, \mathbf{r}^{\prime}, \bar{\rho}\right)\right. \\
& \left.+\rho(\mathbf{r}) \frac{\partial g_{0}\left(\mathbf{r}, \mathbf{r}^{\prime}, \bar{\rho}\right)}{\partial \bar{\rho}}\right] \rho\left(\mathbf{r}^{\prime}\right) u^{a}\left(\mathbf{r}, \mathbf{r}^{\prime}\right) \\
& -\frac{1}{4} \rho^{2}(\mathbf{r}) \frac{\partial}{\partial \rho(\mathbf{r})}\left[\rho(\mathbf{r}) \int d \mathbf{r}^{\prime} \frac{\partial\left[\rho(\mathbf{r}) g_{0}\left(\mathbf{r}, \mathbf{r}^{\prime}, \rho(\mathbf{r})\right)\right]}{\partial p_{0}}\right. \\
& \left.\times\left[u^{a}\left(\mathbf{r}, \mathbf{r}^{\prime}\right)\right]^{2}\right] .
\end{aligned}
$$

For the entropy density per particle, we have

$$
\begin{aligned}
s(\mathbf{r})= & -\left(\frac{\partial}{\partial T} \frac{f(\rho(\mathbf{r}), T)}{\rho(\mathbf{r})}\right)_{\rho(\mathbf{r})} \\
= & s^{0}(\mathbf{r})-\frac{1}{2} \int d \mathbf{r}^{\prime}\left[\frac{\partial g_{0}\left(\mathbf{r}, \mathbf{r}^{\prime}, \bar{\rho}\right)}{\partial T}\right]_{\bar{\rho}} \rho\left(\mathbf{r}^{\prime}\right) u^{a}\left(\mathbf{r}, \mathbf{r}^{\prime}\right) \\
& +\frac{1}{4} \rho(\mathbf{r}) \int d \mathbf{r}^{\prime} \frac{\partial}{\partial T}\left\{\frac{\partial \bar{\rho}}{\partial p_{0}} g_{0}\left(\mathbf{r}, \mathbf{r}^{\prime}, \bar{\rho}\right)\right\} \\
& \rho\left(\mathbf{r}^{\prime}\right) \\
& \times\left[u^{a}\left(\mathbf{r}, \mathbf{r}^{\prime}\right)\right]^{2} .
\end{aligned}
$$

Using the approximation that gave Eq. (27) for the Helmholtz energy, one obtains

$$
\begin{aligned}
s_{I I, A}(\mathbf{r})= & -\left(\frac{\partial}{\partial T} \frac{f_{I I, A}(\rho(\mathbf{r}), T)}{\rho(\mathbf{r})}\right)_{\rho(\mathbf{r})} \\
= & s^{0}(\mathbf{r})-\frac{1}{2} \int d \mathbf{r}^{\prime}\left[\frac{\partial g_{0}\left(\mathbf{r}, \mathbf{r}^{\prime}, \bar{\rho}\right)}{\partial T}\right]_{\bar{\rho}} \rho\left(\mathbf{r}^{\prime}\right) u^{a}\left(\mathbf{r}, \mathbf{r}^{\prime}\right) \\
& +\frac{1}{4} \rho^{2}(\mathbf{r}) \frac{\partial}{\partial T}\left\{\frac{\partial \rho(\mathbf{r})}{\partial p_{0}} \int d \mathbf{r}^{\prime} g_{0}\left(\mathbf{r}, \mathbf{r}^{\prime}, \rho(\mathbf{r})\right)\right. \\
& \left.\times\left[u^{a}\left(\mathbf{r}, \mathbf{r}^{\prime}\right)\right]^{2}\right\} .
\end{aligned}
$$

Using the approximation that gave Eq. (29) for the Helmholtz energy, one obtains

$$
\begin{aligned}
s_{I I, B}(\mathbf{r})= & -\left(\frac{\partial}{\partial T} \frac{f_{I I, B}(\rho(\mathbf{r}), T)}{\rho(\mathbf{r})}\right)_{\rho(\mathbf{r})} \\
= & s^{0}(\mathbf{r})-\frac{1}{2} \int d \mathbf{r}^{\prime}\left[\frac{\partial g_{0}\left(\mathbf{r}, \mathbf{r}^{\prime}, \bar{\rho}\right)}{\partial T}\right]_{\bar{\rho}} \rho\left(\mathbf{r}^{\prime}\right) u^{a}\left(\mathbf{r}, \mathbf{r}^{\prime}\right) \\
& +\frac{1}{4} \rho^{2}(\mathbf{r}) \int d \mathbf{r}^{\prime} \frac{\partial}{\partial T}\left\{\frac{\partial\left[\rho(\mathbf{r}) g_{0}\left(\mathbf{r}, \mathbf{r}^{\prime}, \rho(\mathbf{r})\right)\right]}{\partial p_{0}}\right\}_{\rho(\mathbf{r})} \\
& \times\left[u^{a}\left(\mathbf{r}, \mathbf{r}^{\prime}\right)\right]^{2} .
\end{aligned}
$$

${ }^{1}$ R. Taylor and R. Krishna, Multicomponent Mass Transfer (Wiley, New York, 1993).

${ }^{2}$ V. K. Badam, V. Kumar, F. Durst, and K. Kumar, Exp. Therm. Fluid Sci. 32, 249 (2007).

${ }^{3}$ D. Bedeaux, A. M. Albano, and P. Mazur, Physica A 82, 438 (1976).

${ }^{4}$ D. Bedeaux, Adv. Chem. Phys. 64, 47 (1986).

${ }^{5}$ D. Bedeaux, L. F. J. Hermans, and T. Ytrehus, Physica A 169, 263 (1990).

${ }^{6}$ D. Bedeaux, J. A. M. Smit, L. F. J. Hermans, and T. Ytrehus, Physica A 182, 388 (1992).

${ }^{7}$ D. Bedeaux and S. Kjelstrup, Int. J. Thermodyn. 8, 25 (2005).

${ }^{8}$ Y. P. Pao, Phys. Fluids 14, 306 (1971).

${ }^{9}$ Y. P. Pao, Phys. Fluids 14, 1340 (1971).

${ }^{10}$ J. W. Cipolla, Jr., H. Lang, and S. K. Loyalka, J. Chem. Phys. 61, 69 (1974).

${ }^{11}$ L. D. Koffman, M. S. Plesset, and L. Lees, Phys. Fluids 27, 876 (1984).

${ }^{12}$ A. Røsjorde, S. Kjelstrup, D. Bedeaux, and B. Hafskjold, J. Colloid Interface Sci. 240, 355 (2001).

${ }^{13}$ J.-M. Simon, S. Kjelstrup, D. Bedeaux, and B. Hafskjold, J. Phys. Chem. B 108, 7186 (2004).

${ }^{14}$ J. Xu, S. Kjelstrup, D. Bedeaux, A. Røsjorde, and L. Rekvig, J. Colloid Interface Sci. 299, 452 (2006).

${ }^{15}$ J. Ge, S. Kjelstrup, D. Bedeaux, J. M. Simon, and B. Rousseau, Phys. Rev. E 75, 061604 (2007).

${ }^{16}$ G. Fang and C. A. Ward, Phys. Rev. E 59, 417 (1999).

${ }^{17}$ D. Bedeaux and S. Kjelstrup, Physica A 270, 413 (1999).

${ }^{18}$ D. Bedeaux, E. Johannessen, and A. Røsjorde, Physica A 330, 329 (2003).

${ }^{19}$ E. Johannessen and D. Bedeaux, Physica A 330, 354 (2003).

${ }^{20}$ E. Johannessen and D. Bedeaux, Physica A 336, 252 (2004).

${ }^{21}$ E. Johannessen and D. Bedeaux, Physica A 370, 258 (2006).

${ }^{22}$ J. M. Simon, D. Bedeaux, S. Kjelstrup, J. Xu, and E. Johannessen, J. Phys. Chem. B 110, 18528 (2006).

${ }^{23}$ J. Ge, D. Bedeaux, J. M. Simon, and S. Kjelstrup, Physica A 385, 421 (2007).

${ }^{24}$ P. Hohenberg and W. Kohn, Phys. Rev. B 136, B864 (1964).

${ }^{25}$ R. Evans, Fundamentals of Inhomogeneous Fluids (Dekker, New York, 1992), Chap. 3.

${ }^{26}$ J. Z. Wu, AIChE J. 52, 1169 (2006).

${ }^{27}$ J.-P. Hansen and I. R. McDonald, Theory of Simple Liquids, 3rd ed. (Academic, Amsterdam, 2006).

${ }^{28}$ J. Vrabec, G. K. Kedia, G. Fuchs, and H. Hasse, Mol. Phys. 104, 1509 (2006). 
${ }^{29}$ D. Chandler, J. D. Weeks, and H. C. Anderson, J. Chem. Phys. 54, 5237 (1971).

${ }^{30}$ A. Røsjorde, D. W. Fossmo, D. Bedeaux, S. Kjelstrup, and B. Hafskjold, J. Colloid Interface Sci. 232, 178 (2000).

${ }^{31}$ J. A. Barker and D. Henderson, J. Chem. Phys. 47, 4714 (1967).

${ }^{32}$ N. F. Carnahan and K. E. Starling, J. Chem. Phys. 51, 635 (1969).

${ }^{33}$ Y. Rosenfeld, Phys. Rev. Lett. 63, 980 (1989).

${ }^{34}$ Y.-X. Yu and J. Z. Wu, J. Chem. Phys. 117, 10156 (2002).

${ }^{35}$ R. Roth, R. Evans, A. Lang, and G. Kahl, J. Phys.: Condens. Matter 14, 1206312078 (2002)

${ }^{36}$ D. Henderson and J. A. Barker, Physical Chemistry: An Advanced Trea- tise (Academic, New York, 1971), Vol. IIIA.

${ }^{37}$ C. G. Gray and K. E. Gubbins, Theory of Molecular Fluids (Clarendon, Oxford, 1984), Vol. I.

${ }^{38}$ J. A. Barker and D. Henderson, J. Chem. Phys. 47, 2856 (1967).

${ }^{39}$ M. S. Wertheim, Phys. Rev. Lett. 10, 321 (1963).

${ }^{40}$ E. Thiele, J. Chem. Phys. 39, 474 (1963).

${ }^{41}$ C. Tegeler, R. Span, and W. Wagner, J. Phys. Chem. Ref. Data 28, 779 (1999).

${ }^{42}$ E. W. Lemmon and R. T. Jacobsen, Int. J. Thermophys. 25, 21 (2004).

${ }^{43}$ C. A. Ward and D. Stanga, Phys. Rev. E 64, 051509 (2001). 